\title{
EXISTENCE OF A SOLUTION TO A VECTOR-VALUED ALLEN-CAHN EQUATION WITH A THREE WELL POTENTIAL
}

\author{
MARIEL SAEZ TRUMPER
}

ABStract. In this paper we prove existence of a vector-valued solution $v$ to

$$
\begin{aligned}
-\Delta v+\frac{\nabla_{v} W(v)}{2} & =0, \\
\lim _{r \rightarrow \infty} v(r \cos \theta, r \sin \theta) & =c_{i} \text { for } \theta \in\left(\theta_{i-1}, \theta_{i}\right),
\end{aligned}
$$

where $W: \mathbb{R}^{2} \rightarrow \mathbb{R}$ is non-negative function that attains its minimum 0 at $\left\{c_{i}\right\}_{i=1}^{3}$ and the angles $\theta_{i}$ are determined by the function $W$. This solution is an energy minimizer.

\section{INTRODUCTION}

In this paper we establish existence of a vector-valued solution $v: \mathbb{R}^{2} \rightarrow \mathbb{R}^{2}$ to the following elliptic problem:

$$
\begin{aligned}
-\Delta v+\frac{\nabla_{v} W(v)}{2} & =0 \\
\lim _{r \rightarrow \infty} v(r \cos \theta, r \sin \theta) & =c_{i} \text { for } \theta \in\left(\theta_{i-1}, \theta_{i}\right),
\end{aligned}
$$

where $W: \mathbb{R}^{2} \rightarrow \mathbb{R}$ is positive function with three local minima, given by $\left\{c_{i}\right\}_{i=1}^{3}$, and the angles $\theta_{i}$, with $\theta_{3}=2 \pi+\theta_{0}$, are determined by the potential $W$ (for a more precise description on how these angles are determined we refer the reader to definitions (7) and (8) )

In 22 an analogous result was proved by P. Sternberg when $W$ has two minima. Later on, Bronsard, Gui and Schatzman [6] considered potentials with three minima that were equivariant under the symmetry group of the equilateral triangle. Under these conditions they proved existence of a solution to (1)-(2). The system of equations given by (11) was also studied in [8, but the domains considered were bounded and Neumann boundary condition was imposed. In that paper, under appropriate assumptions over the potential $W$, Flores, Padilla and Tonwaga established the existence of solutions that join the three minima $\left(c_{1}\right.$, $c_{2}$ and $c_{3}$ ); however, no precise description of the triple junction was provided. Recently, potentials with four minima were studied in [11, establishing (under several assumptions over the potential $W$ ) the existence of solutions to (11) that connect all the four wells.

Our interest in this problem is originated in some models of three-boundary motion. Material scientists working on the theory of transition layers have found that the motion of grain boundaries is governed by its local mean curvature (see [15, [16] for example). These models naturally arise as the singular limit of the parabolic Allen-Cahn equation (see [2]). The expected relation between grain boundaries motion and the parabolic Allen-Cahn equation can be described as follows: Consider a positive potential $W: \Omega \subset \mathbb{R}^{n} \rightarrow \mathbb{R}$ with 
a finite number of minima $\left\{c_{i}\right\}_{i=i}^{m}$. Let $u_{\epsilon}: \mathbb{R}^{n} \rightarrow \mathbb{R}^{n}$ be a solution to

$$
\frac{d u_{\epsilon}}{d t}-\Delta u_{\epsilon}+\frac{\nabla_{v} W\left(u_{\epsilon}\right)}{2 \epsilon^{2}}=0 .
$$

As $\epsilon \rightarrow 0$ the solutions $u_{\epsilon}$ will converge almost everywhere to one of the constants $c_{i}$ (see [12, [18]). For every $t$, this creates a partition of $\Omega=\bigcup_{i=1}^{m} \Omega_{i}(t)$, where $\Omega_{i}(t)=$ $\left\{x \in \Omega: u_{\epsilon}(x, t) \rightarrow c_{i}\right.$ as $\left.\epsilon \rightarrow 0\right\}$. The interface between these sets correspond to the grain boundaries, which evolve under its curvature. When $n=2$ and $m=3$ the solution will describe a "three-phase" boundary motion that might present "triple- points", namely points where these 3 boundaries meet. Bronsard and Reitich [7] predicted that at points that are away from the triple points and close to the interface between $c_{i}$ and $c_{j}$ the solutions to (3) should be approximated by $\zeta_{i j}\left(\frac{d_{i j}(x, t)}{\epsilon}\right)$, where $d_{i j}$ is the distance function to this interface and $\zeta_{i j}$ is a solution to the equation

$$
\begin{gathered}
\zeta_{i j}^{\prime \prime}(\lambda)+\frac{\nabla W\left(\zeta_{i j}(\lambda)\right)}{2}=0, . \\
\lim _{\tau \rightarrow-\infty} \zeta_{i j}(\tau)=c_{i}, \lim _{\tau \rightarrow \infty} \zeta_{i j}(\tau)=c_{j} .
\end{gathered}
$$

On the other hand, close to the triple points it is expected that solutions to (3) will behave, after appropriate rescaling, like a solution to (11)-(2). However, the existence of such solution has not been established in the general case before. This is the main goal of this paper.

Based on the previous discussion, in order to match the expected behavior of solutions to (3) near double junctions and the one close to triple junctions, we expect that solutions to (11)-(2) satisfy an extra condition at infinity. Namely, solutions to (11)-(2) should resemble solutions to (4)-(5) near the half-lines of direction $\theta_{i}$. We will implicitly impose this condition throughout the paper. Therefore, we briefly discuss the existence of solutions to (4) -(5) . For potentials with two wells the existence of such curves was proved by P. Sternberg in [22. However, the problem is more subtle when considering arbitrary three-well potentials, even if conditions analogous to the ones imposed in 22 hold. In [1 Alikakos, Betelú and Chen provided some examples of potentials where solutions to (44)-(5) did not exist for certain $i, j$. On the other hand, they also established appropriate conditions under which all these solutions in fact do exist. In what follows we will assume we are in the latter case. Namely we assume the existence of $\zeta_{i j}$ for every $i$ and $j$. This and other technical assumptions on the potential $W$ will be discussed in detail in the following section. At the moment we state the main theorem of this paper:

Theorem 1.1. Let $W: \mathbb{R}^{2} \rightarrow \mathbb{R}$ be a proper $\mathcal{C}^{3}$ function that satisfy

(a) $W$ has only three local minima $c_{1}, c_{2}$ and $c_{3}$ and $W\left(c_{i}\right)=0$;

(b) the matrix $\frac{\partial^{2} W(u)}{\partial u_{i} \partial u_{j}}$ is positive definite at $\left\{c_{i}\right\}_{i=1}^{3}$, that is the minima are non-degenerate.

(c) The hessian of the function $W(u)$ (which we denote by $W^{\prime \prime}$ ) is positive semidefinite for $|u|>K$, where $K>0$ is a fixed real number;

(d) there exist positive constants $K_{1}, K_{2}$ and $m$, and a number $p \geq 2$ such that

$$
K_{1}|u|^{p} \leq W(u) \leq K_{2}|u|^{p} \text { for }|u| \geq m ;
$$

(e) Hypothesis 1 holds (see the next section for a description on this hypothesis). In particular, there are solutions to (4)-(5) for every $i$ and $j$. 
Define

(6) $\Gamma\left(\zeta_{1}, \zeta_{2}\right)=\inf \left\{\int_{0}^{1} W^{\frac{1}{2}}(\gamma(\lambda))\left|\gamma^{\prime}(\lambda)\right| d \lambda: \gamma \in C^{1}\left([0,1], \mathbb{R}^{2}\right), \gamma(0)=\zeta_{1}\right.$ and $\left.\gamma(1)=\zeta_{2}\right\}$

Consider $\left\{\alpha_{i}\right\}_{i=1}^{3} \in[0,2 \pi)$ such that

$$
\frac{\sin \alpha_{1}}{\Gamma\left(c_{2}, c_{3}\right)}=\frac{\sin \alpha_{2}}{\Gamma\left(c_{1}, c_{3}\right)}=\frac{\sin \alpha_{3}}{\Gamma\left(c_{1}, c_{2}\right)}
$$

Then for $\theta_{i} \in[0,2 \pi)$ such that

$$
\alpha_{i}=\theta_{i}-\theta_{i-1}
$$

there is a solution $v$ to (1)-(2). Moreover, there exists a differentiable function $\phi$ satisfying (2) such that for

we have

$$
\mathcal{G}(w)=\int_{\mathbb{R}^{2}}\left(|D w|^{2}+W(w)-|D \phi|^{2}-W(\phi)\right) d x
$$

$$
\begin{array}{r}
\mathcal{G}(v)=\inf \{\mathcal{G}(w): w \in \mathcal{V}\}, \\
\text { for } \mathcal{V}=\left\{w \in C^{1}: \int_{\mathbb{R}^{2}}|D w-D \phi| d x, \int_{\mathbb{R}^{2}}|w-\phi| d x<\infty\right\}
\end{array}
$$

We would like to remark that the function $\phi$ in Theorem 1.1 will be defined explicitly in the coming section (more specifically in sub-section 2.2) and it will capture the behavior at infinity of the solution $u$ to (11)-(2). In the construction of this function Hypothesis (E) is required. Relaxations of this hypothesis are possible, but we will skip them in order to keep the presentation simpler. We also want to point out that, as discussed in [7], the definitions of $\alpha_{i}$ and $\Gamma_{i}$ imply that $\alpha_{1}+\alpha_{2}+\alpha_{3}=2 \pi$ and $\theta_{3}=2 \pi+\theta_{0}$.

Before proceeding to the coming sections, we would like to briefly outline our proof of Theorem 1.1 and its organization through the paper. The basic idea is the following: Let $B_{R}$ denote the ball or radius $R$ and let $v_{R}$ solve equation (11) in $B_{R}$ with Dirichlet boundary condition $\left.v_{R}\right|_{\partial B_{r}}=\phi$ (the function $\phi$ is defined in equation (19) and captures the desired behaviour at infinity, as it is discussed in Remark 2.1 below). The proof of Theorem 1.1 will be equivalent to show convergence of the solutions $v_{R}$ in an appropriate norm.

In order to prove the convergence result we use the following key observation: In the unit ball we define the function

then $u_{R}$ satisfies

$$
u_{R}(x)=v_{R}(R x)
$$

$$
-\Delta u_{R}+\frac{R^{2} \nabla_{v} W\left(u_{R}\right)}{2}=0 \text { for } x \in B_{1}
$$

Hence for $\epsilon=\frac{1}{R}$, the function $u_{\epsilon}$ satisfies

$$
-\Delta u_{\epsilon}+\frac{\nabla_{v} W\left(u_{\epsilon}\right)}{2 \epsilon^{2}}=0
$$

As $R \rightarrow \infty$ (or equivalently as $\epsilon \rightarrow 0$ ) we expect $v_{R}$ to converge to the solution $v$ to (11)-(2) (this will be proved in Section 5), and correspondingly, we expect the limiting solution $u_{\epsilon}$ to (9) to capture the behavior of $v$ at infinity. Equation (9) has been largely studied (see for example [5] and [17]). This motivates us to analyze in Section 3 some existing results for (9) that apply in our context and provide useful information for our problem. More precisely, combining results in [3, [13] and [23] and using $\Gamma$-convergence techniques we prove that the rescaled $u_{\epsilon}$ converge to a function $u_{0}$ in the $L^{1}$ norm in the unit ball. Moreover, the 
function $u_{0}$ equals $c_{i}$ in the the angular sectors defined by $\theta \in\left(\theta_{i-1}, \theta_{i}\right)$ and it is minimizing for an appropriate functional (eventually, this property will imply the minimizing result in Theorem 1.11). Hypotheses (d) and (远) are essential in this section. However, we would like to point out that it is not clear whether they are just technical conditions (which may be removed) or not. On the other hand, hypotheses (国) and (b) (which are also used in this section) are natural in the context of the problem.

In order to finish the proof, in Section 4 we show that the convergence holds in a stronger norm than $L^{1}$. The main idea in this computation is to use the parabolic version of equation (9) to interpolate between an approximate solutions to (11) in the ball (which we will denote by $\left.U_{\vec{q}}\right)$ and the real solution. More precisely, we consider a function $\tilde{h}_{\epsilon}$ that is a solution to

$$
\begin{aligned}
\frac{d \tilde{h}_{\epsilon}}{d t}-\Delta \tilde{h}_{\epsilon}+\frac{\nabla_{v} W\left(\tilde{h}_{\epsilon}\right)}{2 \epsilon^{2}} & =0 \text { for } x \in B_{1}, t \in(0, \infty) \\
\tilde{h}(x, t) & =\phi_{\epsilon}(x) \text { for } x \in \partial B_{1}, \\
\tilde{h}(x, 0) & =U_{\vec{q}} .
\end{aligned}
$$

The "approximate solution" $U_{\vec{q}}$ depends on $\epsilon$, satisfies $U_{\vec{q}}=\phi_{\epsilon}(x)$ for $x \in \partial B_{1}$ and $\left(-\Delta U_{\vec{q}}+\frac{\nabla_{v} W\left(U_{\vec{q}}\right)}{2 \epsilon^{2}}\right)(x) \rightarrow 0$ as $\epsilon \rightarrow 0$ point-wise in $B_{1}$. Using Theorem 4.1 we prove that in fact $\tilde{h}_{\epsilon}$ and $U_{\vec{q}}$ remain appropriately close in time. We conclude by observing that, as $t \rightarrow \infty$ it holds that $\tilde{h}_{\epsilon}(\cdot, t) \rightarrow u_{\epsilon}(\cdot)$. This will imply that in fact $u_{\epsilon}$ is close to $U_{\vec{q}}$. Also in that section, we use similar techniques to control the convergence in compact domains of the sequence $v_{\epsilon}: B_{\frac{1}{\epsilon}} \rightarrow \mathbb{R}^{2}$ given by $v_{\epsilon}(x)=u(\epsilon x)$. The proof of Theorem 1.1 can be easily finished by combining the elements described above. This is achieved in Section 5 .

We would like to remark that the techniques used in this paper were already used in similar problems (see [20] and [21]). In general, the method can be extended as long as the solutions to (11) converge to minima of $W$ as $\epsilon \rightarrow 0$ and that approximate solutions with the desired characteristics (such as $U_{\vec{q}}$ in this case) can be constructed.

The author wishes to thank the referee for the very useful comments in improving the exposition, the Max Planck Institute for Gravitational Physics for providing a great work environment and to Rafe Mazzeo and Alex Freire for very useful discussions.

\section{Definitions AND PRELiminary LEMmas}

We divide this section into three sub-sections. The first one is devoted to several definitions that will be used in the analysis performed in Section 3. The main objective of the second sub-section is to construct the function $\phi$ used in Theorem 1.1. In the final sub-section we summarize a collection of existing results that will be used throughout this paper.

2.1. General definitions. In this sub-section we will address several general definitions that will simplify the notation in the coming section.

Define the function $g_{i}: \mathbb{R}^{2} \rightarrow \mathbb{R}$ for any $p \in \mathbb{R}^{2}$ as

$$
g_{i}(p)=\Gamma\left(c_{i}, p\right)
$$

Where the function $\Gamma$ is defined by (6). Notice that $\Gamma$ can be regarded as degenerate distance function. Hence $g_{i}(p)$ represents the distance of a point $p$ (with respect to the distance function $\Gamma$ ) to the critical point $c_{i}$.

Inspired in [22] we consider the following assuption: 
Hypothesis 1 . Suppose that for every $u \in \mathbb{R}^{2}$, there exists a curve $\gamma_{u}^{i}:[-1,1] \rightarrow \mathbb{R}^{2}$ such that $\gamma_{u}^{i}(-1)=c_{i}, \gamma_{u}^{i}(1)=u$ and

$$
g_{i}(u)=\int_{-1}^{1} \sqrt{W\left(\gamma_{u}^{i}(t)\right)}\left|\left(\gamma_{u}^{i}\right)^{\prime}(t)\right| d t
$$

The function $g_{i}$ is Lipschitz continuous and satisfies

$$
\left|D g_{i}(u)\right|=\sqrt{W(u)} \text { a.e. }
$$

For potentials with two wells the existence of such curves was proved by P. Sternberg in 22. He also proved that when considering a curve that joins the minima of $W$, it can be re-parametrized by a curve $\beta_{i j}:(-\infty, \infty) \rightarrow(-1,1)$ such that the curves defined by

$$
\zeta_{i j}(\tau)=\gamma_{c_{j}}^{i}\left(\beta_{i j}(\tau)\right)
$$

satisfy

$$
2 g_{i}\left(c_{j}\right)=\int_{-\infty}^{\infty} W\left(\zeta_{i j}\right)+\left|\zeta^{\prime}\right|^{2} d \tau
$$

as well as (4) and (5) (where the limits in (5) are attained at an exponential rate). In our situation, if we assume Hypothesis 1, the previous construction can also be carried out. Hence, in what follows we will work under Hypothesis 1 and, in particular, we assume that for any pair of minima $c_{i}, c_{j}$ there is a solution to (4)-(5).

As mentioned in the introduction, we want to relate equation (11)-(2) with the following equation in the unit ball:

$$
\begin{aligned}
-\Delta u_{\epsilon}+\frac{\nabla_{v} W\left(u_{\epsilon}\right)}{\epsilon^{2}} & =0 \text { for } x \in B_{1} \\
\left.u_{\epsilon}\right|_{\partial B_{1}}(x) & =\phi_{\epsilon}(x) .
\end{aligned}
$$

where $\phi_{\epsilon}$ will be properly defined in the coming sub-section. This equation motivates us to define the following functional:

$$
\mathcal{I}_{\epsilon}(u)=\left\{\begin{array}{cl}
\int_{B_{1}} \epsilon|D u|^{2}+\frac{1}{\epsilon} W(u) d y & \text { if } u \in H^{1}\left(B_{1}\right) \text { and }\left.u\right|_{\partial B_{1}}(x)=\phi_{\epsilon}(x) \\
\infty & \text { otherwise. }
\end{array}\right.
$$

where $u: B_{1} \rightarrow \mathbb{R}^{2}, \phi_{\epsilon}: \partial B_{1} \rightarrow \mathbb{R}^{2}$. It is easy to check that weak solutions to (14)-(15) can be regarded as critical points of (16).

We are interested in studying the limiting problem as $\epsilon \rightarrow 0$. More specifically, we expect the limit of the solutions $u_{\epsilon}$ to (14) will capture the behavior at infinity of the function $v$ which satisfies (1)-(2). In particular, we want to show that it is possible to obtain as the limit of the functions $u_{\epsilon}$ a function $u_{0}$ that satisfies

$$
u_{0}(r \cos \theta, r \sin \theta)=c_{i} \text { for } \theta \in\left(\theta_{i-1}, \theta_{i}\right),
$$

where $\alpha_{i}=\theta_{i}-\theta_{i-1}$ satisfy (7). Without loss of generality we are going to assume that $\theta_{0}=0$ and $\theta_{3}=2 \pi$.

In order to study the limit of the functions $u_{\epsilon}$ we define the following limit functional (that we will show corresponds to the $\Gamma$-limit of the functionals $\mathcal{I}_{\epsilon}$ ): 


$$
\mathcal{I}_{0}(u)=\left\{\begin{array}{cc}
\sum_{i, j=1}^{3} \Gamma\left(c_{i}, c_{j}\right) H_{1}\left(\partial_{B_{1}} \Omega_{i}(u) \bigcap \partial_{B_{1}} \Omega_{i+1}(u)\right) & \text { if } g_{i}(u) \in B V\left(B_{1}\right) \\
+\sum_{i, j=1}^{3} \Gamma\left(c_{i}, c_{j}\right) H_{1}\left(\left(\partial \Omega_{j}(u) \bigcap \partial B_{1}\right) \backslash \Phi_{i}\right) & \text { and } u \in\left\{c_{i}\right\}_{i=0}^{3} \\
\infty & \text { otherwise }
\end{array}\right.
$$

where $\Omega_{i}(u)=\left\{x \in B_{1}: u(x)=c_{i}\right\}, \phi_{0}(x)=\lim _{\epsilon \rightarrow 0} \phi_{\epsilon}(x), \Phi_{i}=\left\{x \in \partial B_{1}: \phi_{0}(x)=c_{i}\right\}$ and $H_{1}$ is the one dimensional Hausdorff measure.

2.2. The function $\phi$. As described in the introduction, the function $\phi$ should represent the boundary condition at infinity, that is, it should satisfy (2). In particular, we expect the sequence of functions $\phi_{\epsilon}$ (defined by $\phi_{\epsilon}(x)=\phi\left(\frac{x}{\epsilon}\right)$ ) to converge to $c_{i}$ as $\epsilon \rightarrow 0$ in the angular sectors of $B_{1}$ defined by $\theta \in\left(\theta_{i-1}, \theta_{i}\right)$ (where the angles $\theta_{i}$ are defined by (77)-(8) ). Moreover, we will construct a function $\phi$ that away from the triple point, approximates a solution to (9) (we will make this statement more precise in section 4).

More precisely, let $L_{i}$ be the half-lines starting at the origin, with direction $\theta_{i}$. Away from $L_{i}$, the function $\phi$ is defined by one of the constants $c_{j}$ (that is, one of the minima of $W)$. Notice that in fact $c_{j}$ are solutions to (14). Near the half-lines $L_{i}$, the function $\phi$ will be equal to an appropriate solution to (4) (that we denote $\zeta_{i j}$ ), evaluated at the distance to $L_{i}$. These functions are approximate solutions in the sense to be discussed in section 4 .

We summarize the description above with the following equations: Consider a smooth function $\eta: \mathbb{R}^{2} \rightarrow \mathbb{R}$ such that $\eta(x) \equiv 1$ when $|x| \leq \frac{1}{2}$ and $\eta(x) \equiv 0$ for $|x| \geq 1$, the distance

$$
d_{i}(x)=d\left(x, L_{i}\right)
$$

and a partition of unity $\left\{\eta_{i}\right\}_{i=1}^{6}$ associated to the family of intervals $\left\{\mathcal{A}_{j}\right\}_{j=1}^{6}$, where

$$
\begin{gathered}
\mathcal{A}_{2 i}=\left(\theta_{i}-\delta, \theta_{i}+\delta\right), \\
\mathcal{A}_{2 i+1}=\left(\theta_{i}+\frac{\delta}{2}, \theta_{i+1}-\frac{\delta}{2}\right) .
\end{gathered}
$$

Now we define

$$
\phi(x)=(1-\eta(x))\left(\eta_{5}(\theta) c_{3}+\eta_{6}(\theta) \zeta_{31}\left(d_{0}(x)\right)+\sum_{i=1}^{2}\left(\eta_{2 i}(\theta) \zeta_{i i+1}\left(d_{i}(x)\right)+\eta_{2 i-1}(\theta) c_{i}\right)\right)
$$

and

$$
\phi_{\epsilon}(x)=\phi\left(\frac{x}{\epsilon}\right) .
$$

Notice that since $L_{i}$ is a half-line we have that $d_{i}\left(\frac{x}{\epsilon}\right)=\frac{d_{i}(x)}{\epsilon}$.

Remark 2.1. The functions $\phi_{\epsilon}$ are not only well defined on the boundary of $B_{1}$, but also in the interior. Moreover, under these definitions we have that

$$
\phi_{0}(x):=\lim _{\epsilon \rightarrow 0} \phi_{\epsilon}(x)=u_{0}(x) \text { a.e. }
$$

Furthermore, in Section 4 will be shown that near the boundary (more precisely for $|x|>$ $\left.\epsilon^{\alpha}\right)$ the function $\phi_{\epsilon}$ is an "approximate solution" to the equation (11) (in the sense that for every $x$ holds $\left(-\Delta \phi_{\epsilon}+\frac{\nabla_{v} W(u)}{2 \epsilon^{2}}\right)(x) \rightarrow 0$ as $\epsilon \rightarrow 0$.) We will prove that in fact for every $\alpha<1$ holds $\sup _{\epsilon^{\alpha}<|x|<1}\left|u_{\epsilon}-\phi_{\epsilon}\right| \rightarrow 0$ as $\epsilon \rightarrow 0$. Correspondingly, for $v_{\epsilon}: B_{\frac{1}{\epsilon}} \rightarrow \mathbb{R}^{2}$ defined by $v_{\epsilon}(x)=u_{\epsilon}(\epsilon x)$ holds $\sup _{\epsilon^{\alpha-1}<|x|<\frac{1}{\epsilon}}\left|v_{\epsilon}-\phi\right| \rightarrow 0$ as $\epsilon \rightarrow 0$. 
On the other hand, it is not expected that the functions $\phi_{\epsilon}$ are good approximations to the solution inside the ball of radius $\epsilon^{\alpha}$ (or correspondingly, $\phi$ is not a good approximation of $v_{\epsilon}$ in the ball of radius $\left.\epsilon^{\alpha-1}\right)$. This can be illustrated as follows: The choice of the functions $\phi_{\epsilon}$ in (20) flexible as long as the features described above are preserved (namely, for $|x|>\epsilon^{\alpha}$ they approach $u_{0}$ and they are an approximated solution to the equation). For example, it is possible to consider $\tilde{\phi}_{\epsilon}(x)=\phi\left(\frac{x}{\epsilon}+\ln (\epsilon) x_{0}\right)$. In fact, for every $k \in \mathbb{N}$ holds $\sup _{|x|>\epsilon^{\alpha}}\left|D^{k} \phi_{\epsilon}-D^{k} \tilde{\phi}_{\epsilon}\right| \rightarrow 0$ as $\epsilon \rightarrow 0$. However, for every $\sigma<\epsilon^{1-\alpha}$ we have $\min c_{i}<\sup _{|x|<\epsilon^{\alpha}}\left|\tilde{\phi}_{\epsilon}(x)-\tilde{\phi}_{\sigma}\left(\frac{\sigma x}{\epsilon}\right)\right|$, which contrasts with the second inequality in Theorem 4.1. In particular, it is clear that $\tilde{\phi}$ cannot not be a good approximation of the solution inside the ball of radius $\epsilon^{\alpha}$. Similarly, it is not expected that $\phi_{\epsilon}$ approximates the solution $u_{\epsilon}$ inside the ball of radius $\epsilon^{\alpha}$ (or that the corresponding function $v_{\epsilon}$ would be approximated by $\phi$ inside the ball of radius $\left.\epsilon^{\alpha-1}\right)$.

2.3. Techinal Lemmas. Now we state some technical lemmas. The first one was originally proved in [19]:

Lemma 2.1. Let $u_{\epsilon}(x) \in \mathcal{C}^{2}$ satisfy (14)-(15), where $W: \mathbb{R}^{2} \rightarrow \mathbb{R}$ is a proper function in $\mathcal{C}^{2}$ bounded below, with a finite number of critical points (that we label as $\left\{c_{i}\right\}_{i=1}^{m}$ ), and such that the Hessian of $W(u)$ is positive semidefinite for $|u| \geq K$ for some real number $K$. Suppose that the functions $\phi_{\epsilon}$ are uniformly bounded. Then there is a constant $C$ depending only on uniform bounds over $\phi_{\epsilon}$ and $W$, but not on $\epsilon$, such that

$$
\sup \left|u_{\epsilon}\right| \leq C \text {. }
$$

Proof. Consider $\omega_{\epsilon}(x)=W\left(u_{\epsilon}\right)(x)$; then

$$
\begin{aligned}
-\Delta \omega_{\epsilon} & =-\sum_{i}\left(\nabla_{v} W\left(u_{\epsilon}\right) \cdot\left(u_{\epsilon}\right)_{x_{i}}\right)_{x_{i}} \\
& =-\left(W^{\prime \prime}\left(u_{\epsilon}\right) D u_{\epsilon}\right) \cdot D u_{\epsilon}-\nabla_{v} W\left(u_{\epsilon}\right) \cdot \Delta u_{\epsilon},
\end{aligned}
$$

where $W^{\prime \prime}$ denotes the Hessian matrix of $W$ and the dot product between two $2 \times 2$ matrices is the standard dot product in $\mathbb{R}^{4}$. Since $u_{\epsilon}$ satisfies (14), this becomes

$$
-\Delta \omega_{\epsilon}+\frac{\left|W^{\prime}\left(u_{\epsilon}\right)\right|^{2}}{2 \epsilon^{2}}+\left(W^{\prime \prime}\left(u_{\epsilon}\right) D u\right) \cdot D u_{\epsilon}=0 .
$$

If the maximum of $\omega_{\epsilon}$ is attained at the boundary, then it is bounded by the maximum of $W\left(\phi_{\epsilon}(x)\right)$.

Suppose that $\omega_{\epsilon}$ has an interior maximum at $x_{0}$ and $\left|u_{\epsilon}\left(x_{0}\right)\right| \geq K$. Since $x_{0}$ is a maximum for $\omega_{\epsilon}$, it holds that $\Delta \omega_{\epsilon}\left(x_{0}\right) \leq 0$. We also have by hypothesis that $W^{\prime \prime}(u)$ is positive semidefinite for $|u| \geq K$, hence

$$
-\Delta \omega_{\epsilon}+\frac{\left|D_{u} W\left(u_{\epsilon}\right)\right|^{2}}{\epsilon^{2}}+\left(W^{\prime \prime}\left(u_{\epsilon}\right) D u_{\epsilon}\right) \cdot D u_{\epsilon} \geq 0 .
$$

The inequality is strict (which contradicts (21)) unless

$$
\frac{\left|D_{u} W\left(u_{\epsilon}\right)\right|^{2}}{\epsilon^{2}}=\left(W^{\prime \prime}\left(u_{\epsilon}\right) D u_{\epsilon}\right) \cdot D u_{\epsilon}=0
$$

If $\nabla_{v} W\left(u_{\epsilon}\left(x_{0}\right)\right)=0$, we would have $u_{\epsilon}\left(x_{0}\right)=c_{i}$ for some $i$ and this implies (since the maximum is attained at this point) that $W\left(u_{\epsilon}(x, t)\right) \leq W\left(c_{i}\right)$. Hence we have $\omega_{\epsilon} \leq$ $\max \left\{\sup _{|u| \leq K} W\left(u_{\epsilon}\right), W\left(\phi_{\epsilon}\right), \max _{i=1 \ldots m} W\left(c_{i}\right)\right\}$. 
Since $W$ is a proper function, we conclude the result of the Lemma.

We will also use Lemma A.1 and Lemma A.2 in [4]. We restate them here without proof:

Lemma 2.2. [Lemma A.1 in [4]] Assume that u satisfies

$$
-\Delta u=f \text { on } \Omega \subset \mathbb{R}^{n}
$$

Then

$$
|D u(x)|^{2} \leq C\left(\|f\|_{L^{\infty}(\Omega)}\|u\|_{L^{\infty}(\Omega)}+\frac{1}{\operatorname{dist}^{2}(x, \partial \Omega)}\|u\|_{L^{\infty}(\Omega)}^{2}\right) \quad \forall x \in \Omega,
$$

where $C$ is a constant depending only on $n$.

Lemma 2.3. [Lemma A.2 in [4]]

Assume that $u$ satisfies

$$
\begin{gathered}
-\Delta u=f \text { on } \Omega \subset \mathbb{R}^{n} \\
u=0 \text { on } \partial \Omega
\end{gathered}
$$

where $\Omega$ is a smooth bounded domain. Then it holds

$$
\|D u\|_{L^{\infty}(\Omega)}^{2} \leq C\|f\|_{L^{\infty}(\Omega)}\|u\|_{L^{\infty}(\Omega)}
$$

where $C$ is a constant depending only on $\Omega$.

\section{Convergence in $L^{1}$}

In this section we show that solutions $u_{\epsilon}$ to equation (14)-(15) converge in $L^{1}$. More precisely, we prove the following result

Proposition 3.1. Let $u_{0}$ be defined by (17). Consider $\mathcal{I}_{\epsilon}$ and $\mathcal{I}_{0}$ defined by (16) and (18) respectively. For $\phi_{\epsilon}$ defined by (19)-(20) there exists a sequence of minimizers $u_{\epsilon}$ of $\mathcal{I}_{\epsilon}$, such that $\mathcal{I}_{\epsilon}\left(u_{\epsilon}\right) \rightarrow \mathcal{I}_{0}\left(u_{0}\right)$ and $u_{\epsilon} \rightarrow u_{0}$ in $L^{1}$.

As stated in 23, when considering the Neumman boundary condition problem, Proposition 3.1 follows from results in [3], [13] and [23. In what follows we are going to state these results and point out the necessary modifications in our setting.

Theorem 3.1. (23]) Let $u_{0}$ be defined by (17) and $C_{i}=\left\{x \in \Omega: u_{0}(x)=c_{i}\right\}$. Consider $a$ domain $\Omega$ and partition $(E, F, G)$ of $\Omega$. Define

$$
\begin{gathered}
\mathcal{F}(E, F, G)=\Gamma\left(c_{1}, c_{2}\right) H_{1}\left(\partial_{\Omega} E \bigcap \partial_{\Omega} G\right)+\Gamma\left(c_{1}, c_{3}\right) H_{1}\left(\partial_{\Omega} E \bigcap \partial_{\Omega} F\right) \\
+\Gamma\left(c_{3}, c_{2}\right) H_{1}\left(\partial_{\Omega} F \bigcap \partial_{\Omega} G\right) .
\end{gathered}
$$

Then the partition formed by $C_{1}, C_{2}$ and $C_{3}$ is an isolated local minimizer of $\mathcal{F}$, that is

$$
\mathcal{F}\left(C_{1}, C_{2}, C_{3}\right)=\min \mathcal{F}(E, F, G)
$$

where the minimum is taken over all the partitions $(E, F, G)$ of $\Omega$ satisfying the condition

$$
\left|C_{1} \Delta E\right|+\left|C_{2} \Delta F\right|+\left|C_{3} \Delta G\right| \leq \delta,
$$

where $\delta$ is some small positive number.

Remark 3.1. The proof of Lemma 3.1 in [23] implies that this $\delta$ can be uniformly chosen for balls of all radii. 
Theorem 3.2. (Theorem 2.5 in [3]) Let

$$
\tilde{\mathcal{I}}_{\epsilon, \Omega}(u)=\left\{\begin{array}{cl}
\int_{\Omega} \epsilon|D u|^{2}+\frac{1}{\epsilon} W(u) d y & \text { if } u \in H^{1}(\Omega) \text { and } \int_{\Omega} u(x) d x=m \\
\infty & \text { otherwise. }
\end{array}\right.
$$

and

$\tilde{\mathcal{I}}_{0, \Omega}(u)=\left\{\begin{array}{cc}\sum_{i, j=1}^{3} \Gamma\left(c_{i}, c_{j}\right) H_{1}\left(\partial_{B_{1}} \Omega_{i}(u) \bigcap \partial_{B_{1}} \Omega_{j}(u)\right) & \text { if } g_{i}(u) \in B V(\Omega) \text { for } i \in\{1,2,3\}, \\ & W(u(x))=0 \text { a.e. and } \int_{\Omega} u(x) d x=m \\ \infty & \text { otherwise }\end{array}\right.$

It holds for every $\epsilon_{h} \rightarrow 0$ that

- For every $u_{\epsilon_{h}} \rightarrow u$ in $L^{1}(\Omega)$ we have that $\tilde{\mathcal{I}}_{0}(u) \leq \liminf _{h \rightarrow \infty} \tilde{\mathcal{I}}_{\epsilon_{h}}\left(u_{\epsilon_{h}}\right)$

- There is $u_{\epsilon_{h}} \rightarrow u$ in $L^{1}(\Omega)$ such that $\tilde{\mathcal{I}}_{0}(u) \geq \lim \sup _{h \rightarrow \infty} \tilde{\mathcal{I}}_{\epsilon_{h}}\left(u_{\epsilon_{h}}\right)$

Proposition 3.2. ( Proposition 2.2 in [3]) The function $g_{i}$ is locally Lipschitz-continuous. Moreover, if $u \in H^{1}(\Omega) \cup L^{\infty}(\Omega)$, then $g_{i}(u) \in W^{1,1}(\Omega)$ and the following inequality holds:

$$
\int_{\Omega}\left|D\left(g_{i}(u)\right)\right| d x \leq \int_{\Omega} \sqrt{W(u)}|D u| d x .
$$

Remark 3.2. Following the proof of Theorem 3.2 in [3] it is easy to see that the restriction $\int_{\Omega} u(x) d x=m$, imposed by Baldo in his work, can be removed from Theorem 3.2 without modifying the proof.

Theorem 3.3. 13] Suppose that a sequence of functionals $\left\{\mathcal{I}_{\epsilon}\right\}$ and a functional $\mathcal{I}_{0}$ satisfying the following conditions:

(1) if $w_{\epsilon} \rightarrow w_{0}$ in $L^{1}(\Omega)$ as $\epsilon \rightarrow 0$, then $\liminf \mathcal{I}_{\epsilon}\left(w_{\epsilon}\right) \geq \mathcal{I}_{0}\left(w_{0}\right)$;

(2) for any $w_{0} \in L^{1}(\Omega)$ there is a family $\left\{\rho_{\epsilon}\right\}_{\epsilon>0}$ with $\rho_{\epsilon} \rightarrow w_{0}$ in $L^{1}(\Omega)$ and $\mathcal{I}_{\epsilon}\left(\rho_{\epsilon}\right) \rightarrow$ $\mathcal{I}_{0}\left(w_{0}\right)$

(3) any family $\left\{w_{\epsilon}\right\}_{\epsilon>0}$ such that $\mathcal{I}_{\epsilon}\left(w_{\epsilon}\right) \leq C<\infty$ for all $\epsilon>0$ is compact in $L^{1}(\Omega)$;

(4) there exits an isolated $L^{1}$-local minimizer $u_{0}$ of $\mathcal{I}_{0}$; that is, $\mathcal{I}_{0}\left(u_{0}\right)<\mathcal{I}_{0}(w)$ whenever $0<\left\|u_{0}-w\right\|_{L^{1}(\Omega)} \leq \delta$ for some $\delta>0$.

Then there exits an $\epsilon_{0}>0$ and a family $\left\{u_{\epsilon}\right\}$ for $\epsilon<\epsilon_{0}$ such that $u_{\epsilon}$ is an $L^{1}$-local minimizer of $\mathcal{I}_{\epsilon}$ and $u_{\epsilon} \rightarrow u_{0}$ in $L^{1}(\Omega)$

Theorem 3.2 establish conditions 1 and 2 of Theorem 3.3 for $\tilde{\mathcal{I}}_{\epsilon, \Omega}$ (defined by (26) ) and $\tilde{\mathcal{I}}_{0}$ (defined by (27)). Theorem 3.1 establishes that $u_{0}$ is a local minimizer for $\tilde{\mathcal{I}}_{0, \Omega}$ (condition 4 of Theorem [3.3). We need to show that these theorems imply that the conditions of Theorem 3.3 also hold for $\mathcal{I}_{\epsilon}$ and $\mathcal{I}_{0}$ (defined by (16) and (18), respectively). In addition, we need to prove that condition 3 holds for these functionals.

Lemma 3.1. Theorem 3.1 implies that $u_{0}$ is a local minimizer for $\mathcal{I}_{0}$.

Proof. Let $C_{i}=\left\{x \in B_{1}: u_{0}(x)=c_{i}\right\}$ and for any $w$ let $\Omega_{i}(w)=\left\{x \in B_{1}: w(x)=c_{i}\right\}$. Consider $\delta$ for $B_{1}$ as is Theorem 3.1. We are going to show by contradiction that for every $w$ such that $w(x) \in\left\{c_{i}\right\}_{i=1}^{3}$ almost everywhere and

$$
\left|C_{1} \Delta \Omega_{1}(w)\right|+\left|C_{2} \Delta \Omega_{2}(w)\right|+\left|C_{3} \Delta \Omega_{3}(w)\right| \leq \delta
$$


holds that

$$
\mathcal{I}_{0}\left(u_{0}\right) \leq \mathcal{I}_{0}(w)
$$

Suppose that there is a $w$ such that

$$
\left|C_{1} \Delta \Omega_{1}(w)\right|+\left|C_{2} \Delta \Omega_{2}(w)\right|+\left|C_{3} \Delta \Omega_{3}(w)\right| \leq \delta
$$

and

$$
\mathcal{I}_{0}\left(u_{0}\right)>\mathcal{I}_{0}(w)
$$

Consider $\sigma>0$ and $B_{1+\sigma}$. Define

$$
\mathcal{I}_{\epsilon}^{\sigma}(u)=\tilde{\mathcal{I}}_{\epsilon, B_{1+\sigma}}(u) \text {. }
$$

Notice first that $u_{0}$ (given by (17)) is well defined for every $x \in \mathbb{R}^{2}$. In particular is well defined for every $x \in B_{1+\sigma}$ for any $\sigma>0$. Hence, we can define

$$
w^{\sigma}(x)=\left\{\begin{array}{cc}
w(x) & \text { if } x \in \bar{B}_{1} \\
u_{0}(x) & \text { if } x \in B_{1+\sigma} \backslash B_{1}
\end{array} .\right.
$$

Let

$$
\begin{gathered}
\tilde{C}_{i}=\left\{x \in B_{1+\sigma}: u_{0}(x)=c_{i}\right\} \\
\tilde{\Omega}_{i}(w)=\left\{x \in B_{1+\sigma}: w^{\sigma}(x)=c_{i}\right\} .
\end{gathered}
$$

Using definition (31) and equation (29) we also have

$$
\left|\tilde{C}_{1} \Delta \tilde{\Omega}_{1}(w)\right|+\left|\tilde{C}_{2} \Delta \tilde{\Omega}_{2}(w)\right|+\left|\tilde{C}_{3} \Delta \tilde{\Omega}_{3}(w)\right| \leq \delta .
$$

Notice that every subset on the boundary where $w$ does not agree with $u_{0}$ becomes an interior boundary term for $w^{\sigma}$ in $B_{1+\sigma}$. By the definition of definition $\mathcal{I}_{0}^{\sigma}$ we have that

$$
\mathcal{I}_{0}^{\sigma}\left(w^{\sigma}\right)=\mathcal{I}_{0}\left(w^{\sigma}\right)+\sigma \sum_{i, j=1}^{3} \Gamma\left(c_{i}, c_{j}\right)
$$

and

$$
\mathcal{I}_{0}^{\sigma}\left(u_{0}\right)=\mathcal{I}_{0}\left(u_{0}\right)+\sigma \sum_{i, j=1}^{3} \Gamma\left(c_{i}, c_{j}\right) .
$$

Inequality (30) implies that

$$
\mathcal{I}_{0}^{\sigma}\left(w^{\sigma}\right)<\mathcal{I}_{0}^{\sigma}\left(u_{0}\right)
$$

which together with (32) contradicts the local minimality of $u_{0}$ given by Theorem 3.1 .

Proof of Proposition 3.1. In what follows, we are going to show that Theorem 3.2 and Proposition 3.2 imply conditions 11 and 2 of Theorem 3.3 for the functionals defined by (16) and (18).

Recall that $\phi_{\epsilon}$ is given by (20),$\phi_{0}=\lim _{\epsilon \rightarrow 0} \phi_{\epsilon}$ and $\phi_{0}=u_{0}$ a.e.

Proof of condition 1, Let

$$
w_{\epsilon} \rightarrow w_{0} \text { in } L^{1} .
$$

As in the proof of Lemma 3.1, consider $\sigma>0$ and define

$$
\mathcal{I}_{\epsilon}^{\sigma}(u)=\tilde{\mathcal{I}}_{\epsilon, B_{1+\sigma}}(u)
$$




$$
w_{\epsilon}^{\sigma}(x)=\left\{\begin{array}{cc}
w_{\epsilon}(x) & \text { if } x \in \bar{B}_{1} \\
\phi_{\epsilon}(x) & \text { if } x \in B_{1+\sigma} \backslash B_{1}
\end{array} .\right.
$$

and

$$
w_{0}^{\sigma}(x)=\left\{\begin{array}{cc}
w_{0}(x) & \text { if } x \in \bar{B}_{1} \\
\phi_{0}(x) & \text { if } x \in B_{1+\sigma} \backslash B_{1}
\end{array} .\right.
$$

Notice that again the boundary portions of $w_{0}$ that do not agree with $\phi_{0}$ become interior boundaries of $w_{\sigma}^{0}$. Hence, as before, if $\mathcal{I}_{0}^{\sigma}\left(w_{0}\right) \neq \infty$ we have that

$$
\mathcal{I}_{0}^{\sigma}\left(w_{0}\right)=\mathcal{I}_{0}\left(w_{0}\right)+\sigma \sum_{i, j=1}^{3} \Gamma\left(c_{i}, c_{j}\right) .
$$

Using (34) and definitions (36) and (37) we have that

$$
w_{\epsilon}^{\sigma} \rightarrow w_{0}^{\sigma} \text { in } L^{1} .
$$

Theorem 3.2 and Remark 3.2 imply that

$$
\mathcal{I}_{0}^{\sigma}\left(w_{0}^{\sigma}\right) \leq \liminf _{\epsilon \rightarrow 0} \mathcal{I}_{\epsilon}^{\sigma}\left(w_{\epsilon}^{\sigma}\right)
$$

We can explicitly compute that

$$
\tilde{\mathcal{I}}_{\epsilon, B_{1+\sigma} \backslash B_{1}}\left(\phi_{\epsilon}\right) \rightarrow \sigma \sum_{i, j=1}^{3} \Gamma\left(c_{i}, c_{j}\right) .
$$

It is also easy to check that

$$
\mathcal{I}_{\epsilon}^{\sigma}\left(w_{\epsilon}\right)=\mathcal{I}_{\epsilon}\left(w_{\epsilon}\right)+\tilde{\mathcal{I}}_{\epsilon, B_{1+\sigma} \backslash B_{1}}\left(\phi_{\epsilon}\right) .
$$

Equations (401) and (41) imply that

$$
\mathcal{I}_{\epsilon}^{\sigma}\left(w_{\epsilon}\right) \rightarrow \infty \text { if and only if } \mathcal{I}_{\epsilon}\left(w_{\epsilon}\right) \rightarrow \infty .
$$

We can assume that $\liminf _{\epsilon \rightarrow 0} \mathcal{I}_{\epsilon}\left(w_{\epsilon}\right)<\infty$ (otherwise the result is trivial). Equations (38), (39), (41) and (40) imply that

$$
\begin{aligned}
\mathcal{I}_{0}\left(w_{0}\right)+\sigma \sum_{i, j=1}^{3} \Gamma\left(c_{i}, c_{j}\right) & =\mathcal{I}_{0}^{\sigma}\left(w_{0}\right) \\
& \leq \liminf _{\epsilon \rightarrow 0} \mathcal{I}_{\epsilon}^{\sigma}\left(w_{\epsilon}^{\sigma}\right) \\
& =\liminf _{\epsilon \rightarrow 0} \mathcal{I}_{\epsilon}\left(w_{\epsilon}\right)+\sigma \sum_{i, j=1}^{3} \Gamma\left(c_{i}, c_{j}\right) .
\end{aligned}
$$

This implies

$$
\mathcal{I}_{0}\left(w_{0}\right) \leq \liminf _{\epsilon \rightarrow 0} \mathcal{I}_{\epsilon}\left(w_{\epsilon}\right)
$$

which proves the result.

Proof of condition [2. The proof of condition 2 follows directly from the proof in [3] of the equivalent statement. Hence, we are going to follow Baldo's proof, use some of his constructions and point out the necessary modifications in our setting. For more details, we refer the reader to 3 . 
As in the proof of condition 1, let $\mathcal{I}_{\epsilon}^{\sigma}$ be defined by (35), that is

$$
\mathcal{I}_{\epsilon}^{\sigma}(u)=\tilde{\mathcal{I}}_{\epsilon, B_{1+\sigma}}(u) .
$$

Consider $w_{0} \in\left\{c_{i}\right\}_{i=1}^{3}$, such that $\mathcal{I}_{0}\left(w_{0}\right)<\infty$ (otherwise the result is trivial). As before, we extend the domain to $B_{1+\sigma}$, for some $\sigma>0$, and we extend $w_{0}$ by $\phi_{0}$ outside the unit ball. We label this extension as $w_{0}^{\sigma}$.

Let $\rho_{\epsilon}^{\sigma}$ be the sequence of functions given by Theorem 3.2 that satisfy $\rho_{\epsilon}^{\sigma} \rightarrow w_{0}^{\sigma}$ in $L^{1}$ and $\mathcal{I}_{\epsilon}^{\sigma}\left(\rho_{\epsilon}\right) \rightarrow \mathcal{I}_{0}^{\sigma}\left(w_{0}^{\sigma}\right)$.

We can write $w_{0}=\sum_{i=1}^{3} c_{i} 1_{\Omega_{i}}$. The functions $\rho_{\epsilon}^{\sigma}$ constructed by Baldo in [3] are uniformly bounded functions, that $\epsilon$ - near the boundaries $\partial \Omega_{i} \bigcap \partial \Omega_{j} \bigcap B_{1+\sigma}$ are equal to the geodesic $\zeta_{i j}$. In the interior of $\Omega_{i}, \rho_{\epsilon}^{\sigma}$ approaches $c_{i}$ uniformly. In particular, we have that $\rho_{\epsilon} \rightarrow w_{0}$ almost everywhere and it is uniformly bounded. By dominated convergence theorem we have that the restriction of $\rho_{\epsilon}^{\sigma}$ to $B_{1}$, that we will label as $\rho_{\epsilon}$, converges to $w_{0}$ in the $L^{1}$ norm.

As in the proof of 1 , we have

$$
\mathcal{I}_{0}^{\sigma}\left(w_{0}^{\sigma}\right)=\mathcal{I}_{0}\left(w_{0}\right)+\sigma \sum_{i, j=1}^{3} \Gamma\left(c_{i}, c_{j}\right) .
$$

By the definitions of $\mathcal{I}_{\epsilon}^{\sigma}, \mathcal{I}_{\epsilon}, \rho_{\epsilon}^{\sigma}$ and $\rho_{\epsilon}$, for every $\sigma>0$ holds that

$$
\mathcal{I}_{\epsilon}^{\sigma}\left(\rho_{\epsilon}^{\sigma}\right) \geq \mathcal{I}_{\epsilon}\left(\rho_{\epsilon}\right)
$$

Combining (42), (43) and Theorem 3.2 we have

$$
\begin{aligned}
\mathcal{I}_{0}\left(w_{0}\right)+\sigma \sum_{i, j=1}^{3} \Gamma\left(c_{i}, c_{j}\right) & =\mathcal{I}_{0}^{\sigma}\left(w_{0}^{\sigma}\right) \\
& =\lim _{\epsilon \rightarrow 0} \mathcal{I}_{\epsilon}^{\sigma}\left(\rho_{\epsilon}^{\sigma}\right) \\
& \geq \lim _{\epsilon \rightarrow 0} \mathcal{I}_{\epsilon}\left(\rho_{\epsilon}\right) .
\end{aligned}
$$

Taking $\sigma \rightarrow 0$ follows that

$$
\mathcal{I}_{0}\left(w_{0}\right) \geq \lim _{\epsilon \rightarrow 0} \mathcal{I}_{\epsilon}\left(\rho_{\epsilon}\right) .
$$

Combining this equation and Condition 1 (that we proved above) we conclude that

$$
\mathcal{I}_{0}\left(w_{0}\right)=\lim _{\epsilon \rightarrow 0} \mathcal{I}_{\epsilon}\left(\rho_{\epsilon}\right),
$$

which finishes the proof.

Proof of condition 3. We will follow the proof in [22]. Suppose that $\mathcal{I}_{\epsilon}\left(w_{\epsilon}\right) \leq C<\infty$ for some family $\left\{w_{\epsilon}\right\}_{\epsilon>0}$.

Define

$$
G_{\epsilon}(x)=g_{1}\left(w_{\epsilon}(x)\right)
$$


Proposition 3.2 implies that

$$
\begin{aligned}
\int_{B_{1}}\left|D G_{\epsilon}(x)\right| d x & \leq \int_{B_{1}} \sqrt{W\left(w_{\epsilon}\right)}\left|D w_{\epsilon}\right| d x \\
& \leq \epsilon \int_{B_{1}}\left|D w_{\epsilon}\right|^{2} d x+\frac{1}{\epsilon} \int_{B_{1}} W\left(w_{\epsilon}\right) d x \\
& \leq C .
\end{aligned}
$$

Hypothesis (d) of Theorem 1.1 implies that $w_{\epsilon}$ are uniformly bounded in $L^{p}\left(B_{1}\right)$ for some $p$. Hence, $G_{\epsilon}$ are uniformly bounded in $L^{1}\left(B_{1}\right)$ and

$$
\left\|G_{\epsilon}\right\|_{B V\left(B_{1}\right)} \leq C \text {. }
$$

Since bounded sequences in BV are compact in $L^{1}([9])$, there is a subsequence $G_{\epsilon}$ convergent to $G_{0}$ in $L^{1}$. This function $G_{0}$ takes the form

$$
G_{0}(x)=\left\{\begin{array}{cc}
0 & \text { if } x \in C_{1} \\
g_{1}\left(c_{2}\right) & \text { if } x \in C_{2} \\
g_{1}\left(c_{3}\right) & \text { if } x \in C_{3} .
\end{array}\right.
$$

Since $c_{1}$ is the only value $x$ such that $g_{1}(x)=0$ and $g_{1}$ is continuous, we have that there is a subsequence $\left\{w_{\epsilon_{j}}\right\}$ that converges in measure to $c_{1}$ on $C_{1}$. The uniform bounds in $L^{p}$ (provided by hypothesis (dd) imply that $\left\{w_{\epsilon_{j}}\right\}$ converge on $C_{1}$ also in the $L^{1}$ norm. The proof can be finished by repeating the same argument for $g_{2}$ and $g_{3}$.

Since Lemma 3.1 implies condition (44) of Theorem 3.3, using that theorem we conclude the result of Proposition 3.1 .

From Theorem 3.3 we conclude the following corollary:

Corollary 3.1. Let $u_{0}$ be defined as in Theorem 3.3. Then there is a subsequence of the family $\left\{u_{\epsilon}\right\}$ that converges point-wise almost everywhere to $u_{0}$.

\section{Uniform Convergence}

In this section we focus on improving the convergence bounds proved in the previous section. Namely, we prove

Theorem 4.1. Fix $0<\alpha<1$. Let $0<\sigma \leq \epsilon^{1-\alpha}$ then for every $m>0$ there is a constant $C$ (that might depend on $\alpha$ and $m$ ) such that

$$
\begin{gathered}
\sup _{|x| \geq \epsilon^{\alpha}}\left|u_{\epsilon}-\phi_{\epsilon}\right| \leq C \epsilon^{m} . \\
\sup _{|x| \leq \frac{\epsilon^{\alpha}}{2}}\left|u_{\epsilon}(x)-u_{\sigma}\left(\frac{\sigma x}{\epsilon}\right)\right| \leq C \epsilon^{m} .
\end{gathered}
$$

There are two main ingredients in the proof of this theorem. The first is the construction of a function $U_{\vec{q}}$ that satisfies $U_{\vec{q}}(x)=\phi_{\epsilon}(x)$ for $x \in B_{1} \backslash B_{\epsilon^{\alpha}}, U_{\vec{q}}(x)=u_{\epsilon}\left(\frac{\sigma x}{\epsilon}\right)$ for $x \in B_{\frac{\epsilon^{\alpha}}{2}}$ and $\left|-\Delta U_{\vec{q}}+\frac{\nabla_{v} W\left(U_{\vec{q}}\right)}{2 \epsilon^{2}}\right|(x) \rightarrow 0$ point-wise; the second one is Theorem 4.2. The idea is the following: We consider $U_{\vec{q}}$ as the initial condition for the parabolic equation (3) in the unit ball. Since $U_{\vec{q}}$ is almost a solution to this equation, we expect that the actual solution to (3) will stay close $U_{\vec{q}}$. This assertion it is ensured by Theorem 4.2, However, in order to apply 
that theorem is necessary to consider solutions to an equation with 0 boundary condition. For this reason, instead of considering equation (3) we take (58)-(59)-(60) (which correspond to subtract the function $U_{\vec{q}}$ from the solution to (3i) ). We finally conclude Theorem 4.1 by observing that our solution to (3) converge to $u_{\epsilon}$ as $t \rightarrow \infty$.

We would also like to remark that the minimizing property of solutions $u_{\epsilon}$ will not be used in this section. In fact, the construction presented here would work for any type of critical point of the functional $\mathcal{I}_{\epsilon}$ with the appropriate boundary values. However, the minimizing property will be used again in section 5 in order to show the minimizing statement of Theorem 1.1

Now we proceed with the construction of the function $U_{\vec{q}}$. Since this functions depends also from other parameters besides $\epsilon$ (such as $\alpha$ above and $\sigma$, which will be shortly introduced) the subindex $\vec{q}$ stands for $\vec{q}=(\epsilon, \sigma, \alpha)$.

Let

$$
\begin{gathered}
v_{\epsilon}(x)=u_{\epsilon}(\epsilon x) \text { and, } \\
u_{\sigma}^{\epsilon}(x)=u_{\sigma}\left(\frac{\sigma x}{\epsilon}\right) .
\end{gathered}
$$

Consider a positive function $\eta: \mathbb{R} \rightarrow \mathbb{R}$ such that $\eta(x)=0$ for $|x| \leq \frac{1}{2}$ and $\eta(x)=1$ for $|x| \geq 1$. Fix $0<\alpha<1$ and

$$
E=2 \epsilon^{\alpha}-\epsilon^{2 m+4-\alpha}
$$

Then define for $y \in \mathbb{R}^{2}$ the function

$$
\eta_{\alpha}(y)=\eta\left(\frac{\epsilon}{2 E}|y|+1-\frac{\epsilon^{\alpha}}{2 E}\right) .
$$

Notice that the function $\eta_{\alpha}(y)$ satisfies $\eta_{\alpha}(y)=0$ for $|y| \leq \epsilon^{\alpha-1}-\frac{E}{\epsilon}$ and $\eta_{\alpha}(x)=1$ for $|y| \geq \epsilon^{\alpha-1}$. Moreover, defining

$$
\eta_{\alpha}^{\epsilon}(y)=\eta_{\alpha}\left(\frac{y}{\epsilon}\right)
$$

it satisfies $\eta_{\alpha}^{\epsilon}(y)=0$ for $|y| \leq \epsilon^{\alpha}-E$ (where $E$ is defined by (44)) and $\eta_{\alpha}^{\epsilon}(y)=1$ for $|y| \geq \epsilon^{\alpha}$.

We will denote by $\mathcal{H}_{\Omega}$ the heat Kernel in $\Omega \subset \mathbb{R}^{2}$. A more detailed description and some properties of the Heat Kernel can be found in the Appendix.

Let

$$
\mathcal{Q}=\left\{(\epsilon, \sigma, \alpha) \in(0,1] \times(0,1] \times[0,1]: \quad \sigma \leq \epsilon^{1-\alpha}\right\} .
$$

Define for $\vec{q}=(\epsilon, \sigma, \alpha) \in \mathcal{Q}$ the function

$$
V_{\vec{q}}(y)=\eta_{\alpha}(y) \phi(y)+\left(1-\eta_{\alpha}(y)\right) v_{\sigma}(y) .
$$

Now we take

$$
U_{\vec{q}}(y)=V_{\vec{q}}\left(\frac{y}{\epsilon}\right) .
$$

Let us denote by $\mathcal{C}_{S}$ the set of continuous functions from $S$ to $\mathbb{R}^{2}$. For $\vec{q}$ as above consider the function $F_{\vec{q}}: \mathcal{C}_{B_{1} \times[0, T]} \times \mathcal{C}_{B_{1}} \rightarrow \mathcal{C}_{B_{1} \times[0, T]}$ defined by

$$
\begin{gathered}
F_{\vec{q}}(h, \psi)(x, t)=\int_{0}^{t} \int_{B_{\frac{1}{\epsilon}}} \mathcal{H}_{B_{\frac{1}{\epsilon}}}(x, y, t-s)\left(-\nabla_{v} W\left(h+V_{\vec{q}}\right)(y, s)+\Delta V_{\vec{q}}\right) d y d s \\
+\int_{B_{\frac{1}{\epsilon}}} \mathcal{H}_{B_{\frac{1}{\epsilon}}}(x, y, t) \psi(y) d y .
\end{gathered}
$$


Notice that, for a given $\psi$, Duhamel's formula implies that, if there is a fixed point $h_{\vec{q}, \psi}$ of $F_{\vec{q}}(\cdot, \psi)$, it would satisfy

$$
\begin{aligned}
\frac{d h_{\vec{q}, \psi}}{d t}-\Delta h_{\vec{q}, \psi}+\frac{\nabla_{v} W\left(h_{\vec{q}, \psi}+V_{\vec{q}}\right)}{2} & =\Delta V_{\vec{q}} \text { in } B_{\frac{1}{\epsilon}} \\
h(x, t) & =0 \text { on } \partial B_{\frac{1}{\epsilon}} \\
h(x, 0) & =\psi(x) .
\end{aligned}
$$

The next lemma shows the existence of such a fixed point:

Lemma 4.1. Fix a uniformly bounded continuous function $\psi_{\epsilon}$ and $\vec{q} \in \mathcal{Q}$, where $\mathcal{Q}$ is defined by (45). The function $F_{\vec{q}}(\cdot, \psi): \mathcal{C}_{B_{1} \times[0, T]} \rightarrow \mathcal{C}_{B_{1} \times[0, T]}$ has a unique fixed point that we label $h_{\vec{q}, \psi}$. Moreover, for $K>0$ and functions $w_{\vec{q}}$ satisfying $\left|w_{\vec{q}}\right| \leq K$, there are constants $M$ and $\beta$ (that might depend on $K$ ), such that for every $T \geq 0$ holds

$$
\begin{aligned}
\sup _{B_{\frac{1}{\epsilon}} \times\left[T, T+\frac{2 \beta}{M}\right]}\left|w_{\vec{q}}-h_{\vec{q}, \psi}\right| \leq \frac{1}{1-\beta}( & 2 \sup _{B_{\frac{1}{\epsilon}} \times\left[T, T+\frac{2 \beta}{M}\right]}\left|F_{\vec{q}}\left(w_{\vec{q}}, \psi\right)-w_{\vec{q}}\right| \\
& \left.+\sup _{x \in B_{\frac{1}{\epsilon}}}\left|w_{\vec{q}}-h_{\vec{q}, \psi}\right|(x, T)\right) .
\end{aligned}
$$

We postpone the proof of this Lemma to the Appendix.

From Lemma 4.1 we can prove the following theorem (which provides one of the essential tools in the proof of Theorem 4.1):

Theorem 4.2. Under the hypothesis of Lemma 4.1, one of the two following alternatives hold:

(1) $\lim _{n \rightarrow \infty} \sup _{B_{\frac{1}{\epsilon}} \times\left[0, T_{n}\right]}\left|w_{n}-h_{\vec{q}_{n}, \psi_{n}}\right| \rightarrow 0$, or

(2) there is a constant $C$, independent of $\vec{q}_{n}$ and $T_{n}$ such that

$$
\sup _{B_{\frac{1}{\epsilon}} \times\left[0, T_{n}\right]}\left|w_{n}-h_{\vec{q}_{n}, \psi_{n}}\right| \leq C \sup _{B_{\frac{1}{\epsilon}} \times\left[0, T_{n}\right]}\left|F_{\vec{q}_{n}}\left(w_{n}, \psi_{n}\right)-w_{n}\right| .
$$

Remark 4.1. Notice that in Theorem 4.2 it is possible to choose $T_{n}=\infty$ for every $n$.

Proof of Theorem 4.2. Consider sequences of continuous functions $\psi_{n}, w_{n}$ satisfying $\sup _{B_{1}}\left|\psi_{n}\right|, \sup _{B_{1} \times\left[0, T_{n}\right)}\left|w_{n}\right| \leq K$ and $\vec{q}_{n} \in \mathcal{Q}$. Suppose that neither (11) nor (2) hold. Then there are subsequences such that

$$
\begin{gathered}
\lim _{n \rightarrow \infty} \sup _{B_{\frac{1}{\epsilon_{n}}} \times\left[0, T_{n}\right]}\left|w_{n}-h_{\vec{q}_{n}, \psi_{n}}\right| \not \rightarrow 0 \text { and, } \\
\sup _{B_{\frac{1}{\epsilon_{n}}} \times\left[0, T_{n}\right]}\left|w_{n}-h_{\vec{q}_{n}, \psi_{n}}\right|=n \sup _{B_{\frac{1}{\epsilon_{n}}} \times\left[0, T_{n}\right]}\left|F_{\vec{q}_{n}}\left(w_{n}, \psi_{n}\right)-w_{n}\right| .
\end{gathered}
$$

The a priori bounds shown in Theorem A-2 and the boundedness hypothesis imply that there is a constant independent of $n$ such that $\left|w_{n}-h_{\vec{q}_{n}, \psi_{n}}\right| \leq C$. Then, (52) implies

$$
\sup _{B_{\frac{1}{\epsilon_{n}}} \times\left[0, T_{n}\right]}\left|F_{\vec{q}_{n}}\left(w_{n}, \psi_{n}\right)-w_{n}\right| \rightarrow 0 .
$$


Applying inequality (49) recursively we have that for every $0 \leq T<\infty$ there is a constant that depends on $T$ (but independent of $\vec{q}_{n}$ ) such that

$$
\sup _{B_{\frac{1}{\epsilon_{n}}} \times[0, T]}\left|w_{n}-h_{\vec{q}_{n}, \psi_{n}}\right| \leq C(T) \sup _{B_{\frac{1}{\epsilon_{n}}} \times[0, T]}\left|F_{\vec{q}_{n}}\left(w_{n}, \psi_{n}\right)-w_{n}\right| .
$$

Therefore if the $T_{n}$ are unifromly bounded, case (2) holds trivially, which contradicts (52).

Hence we may assume $T_{n} \rightarrow \infty$. We will show that in this case

$$
\lim _{n \rightarrow \infty} \sup _{\frac{1}{\epsilon_{n}} \times\left[0, T_{n}\right]}\left|w_{n}-h_{\vec{q}_{n}, \psi_{n}}\right| \rightarrow 0
$$

contradicting a (51).

Let

$$
\tau=\left\{\left(S_{n}\right)_{n \in \mathbb{N}}: 0 \leq S_{n} \leq T_{n}, \lim _{n \rightarrow \infty} \sup _{B_{\frac{1}{\epsilon_{n}}} \times\left[0, S_{n}\right]}\left|w_{n}-h_{\vec{q}_{n}, \psi_{n}}\right| \rightarrow 0\right\} .
$$

For the set of sequences in $\mathbb{R}_{+}$we consider the topology defined by the basis of open sets given by $B_{\sigma}\left(\left(S_{n}\right)_{n \in \mathbb{N}}\right)=\left\{\left(\tilde{S}_{n}\right)_{n \in \mathbb{N}}: \quad \tilde{S}_{n} \geq 0\right.$ and $\left.\sup _{n \in \mathbb{N}}\left|S_{n}-\tilde{S}_{n}\right| \leq \sigma\right\}$ for any $\sigma>0$. Notice that in particular inequality (54) implies that $\tau$ is a non-empty set, since at least $S_{n}=\inf _{n} T_{n} \in \tau$.

\section{Claim: $\tau$ is open}

Consider $\left(S_{n}\right)_{n} \in \tau$. Let $\tilde{S}_{n}=\min \left\{S_{n}+\frac{2 \beta}{M}, T_{n}\right\}$. Using inequality (49) we have $\sup _{B_{\frac{1}{\epsilon_{n}}} \times\left[S_{n}, \tilde{S}_{n}\right]}\left|w_{n}-h_{\vec{q}_{n}, \psi_{n}}\right| \leq \frac{1}{1-\beta}\left(2 \sup _{B_{\frac{1}{\epsilon_{n}}} \times\left[S_{n}, \tilde{S}_{n}\right]}\left|F_{\vec{q}_{n}}\left(w_{n}, \psi_{n}\right)-w_{n}\right|+\sup _{x \in B_{\frac{1}{\epsilon_{n}}}}\left|w_{n}-h_{\vec{q}_{n}, \psi_{n}}\right|\left(x, S_{n}\right)\right)$.

Since $\tilde{S}_{n} \leq T_{n}$ and $S_{n} \in \tau$, taking $n \rightarrow \infty$ we have that

$$
\lim _{n \rightarrow \infty} \sup _{\frac{1}{\epsilon_{n}} \times\left[S_{n}, \tilde{S}_{n}\right]}\left|w_{n}-h_{\vec{q}_{n}, \psi_{n}}\right|=0,
$$

and $B_{\frac{2 \beta}{M}} \bigcap \tau \subset \tau$. Hence $\tau$ is open.

\section{Claim: $\tau$ is closed}

Suppose that $S^{k}=\left(S_{n}^{k}\right)_{n} \in \tau$ satisfy $S^{k} \rightarrow \tilde{S}=\left(\tilde{S}_{n}\right)_{n}$ as $k \rightarrow \infty$. By the definition of the topology we have that there is a $k_{0}$ such that for every $n \in \mathbb{N}$ and $k \geq k_{0}$ holds $\left|S_{n}^{k}-\tilde{S}_{n}\right| \leq \frac{2 \beta}{M}$. Using inequality (49)

$\sup _{B_{\frac{1}{\epsilon_{n}}} \times\left[S_{n}^{k_{0}}, \tilde{S}_{n}\right]}\left|w_{n}-h_{\vec{q}_{n}, \psi_{n}}\right| \leq \frac{1}{1-\beta}\left(2 \sup _{B_{\frac{1}{\epsilon_{n}}} \times\left[S_{n}^{k_{0}}, \tilde{S}_{n}\right]}\left|F_{\vec{q}_{n}}\left(w_{n}, \psi_{n}\right)-w_{n}\right|+\sup _{x \in B_{\frac{1}{\epsilon_{n}}}}\left|w_{n}-h_{\vec{q}_{n}, \psi_{n}}\right|\left(x, S_{n}^{k_{0}}\right)\right)$.

Using that $\left(S_{n}^{k_{0}}\right)_{n} \in \tau$ and (53), when $n \rightarrow \infty$ we have

$\lim _{n \rightarrow \infty} \sup _{B_{\frac{1}{\epsilon_{n}}} \times\left[0, \tilde{S}_{n}\right]}\left|w_{n}-h_{\vec{q}_{n}, \psi_{n}}\right|=\max \left\{\sup _{B_{\frac{1}{\epsilon_{n}}} \times\left[0, S_{n}^{k_{0}}\right]}\left|w_{n}-h_{\vec{q}_{n}, \psi_{n}}\right|, \sup _{B_{\frac{1}{\epsilon_{n}}} \times\left[S_{n}^{k_{0}}, \tilde{S}_{n}\right]}\left|w_{n}-h_{\vec{q}_{n}, \psi_{n}}\right|\right\} \rightarrow 0$.

Therefore $\tilde{S} \in \tau$ and $\tau$ is closed.

Since $\tau$ is open, closed and non-empty we conclude that $\tau=\left\{\left(S_{n}\right)_{n \in \mathbb{N}}: 0 \leq S_{n} \leq T_{n}\right\}$.

In particular $\left(T_{n}\right)_{n} \in \tau$, which contradicts (51) and proves the Theorem. 
Following the proof of Theorem 4.2 we obtain:

Corollary 4.1. Consider the sequences $\psi_{n}, w_{n}, \vec{q}_{n} \in \mathcal{Q}$ and $T_{n}>0$ as in Theorem 4.2. Assume in addition that there are constants $C, m$ such that $\sup _{B_{\frac{1}{\epsilon_{n}}}} \times\left[0, T_{n}\right] \mid F_{\vec{q}_{n}}\left(w_{n}, \psi_{n}\right)-$ $w_{n} \mid \leq C \epsilon_{n}^{m}$. Then for every $\tilde{m}<m$ holds either

(1) $\lim _{n \rightarrow \infty} \sup _{B} \frac{1}{\epsilon_{n}} \times\left[0, T_{n}\right] \frac{\left|w_{n}-h_{\vec{q}_{n}, \psi_{n}}\right|}{\epsilon_{n}^{\dot{m}}} \rightarrow 0$, or

(2) there is a constant $C$, independent of $\vec{q}_{n}$ and $T_{n}$ such that

$$
\sup _{B_{\frac{1}{\epsilon_{n}}} \times\left[0, T_{n}\right]} \frac{\left|w_{n}-h_{\vec{q}_{n}, \psi_{n}}\right|}{\epsilon^{\tilde{m}}} \leq C \sup _{B_{\frac{1}{\epsilon_{n}}} \times\left[0, T_{n}\right]} \frac{\left|F_{\vec{q}_{n}}\left(w_{n}, \psi_{n}\right)-w_{n}\right|}{\epsilon_{n}^{\tilde{m}}} .
$$

In particular, there is a constant $C$ such that

$$
\sup _{\frac{1}{\epsilon_{n}} \times\left[0, T_{n}\right]}\left|w_{n}-h_{\vec{q}_{n}, \psi_{n}}\right| \leq C \epsilon_{n}^{\tilde{m}} .
$$

Now we would like to rescale the estimates of the previous Theorem and Corollary to the unit ball. Namely, instead of considering the function $h_{\vec{q}, \psi_{\epsilon}^{\epsilon}}: B_{\frac{1}{\epsilon}} \times\left[0, \frac{T}{\epsilon^{2}}\right] \rightarrow \mathbb{R}^{2}$ we define the function $k_{\vec{q}, \psi_{\epsilon}^{\epsilon}}: B_{1} \times[0, T] \rightarrow \mathbb{R}^{2}$ by

$$
k_{\vec{q}, \psi_{\epsilon}^{\epsilon}}(x, t)=h_{\vec{q}}\left(\frac{x}{\epsilon}, \frac{y}{\epsilon^{2}}\right) .
$$

Notice that under this definition for every $\epsilon>0$ we can write the left hand side of equation (50) as

$$
\sup _{B_{\frac{1}{\epsilon}} \times\left[0, \frac{T}{\epsilon^{2}}\right]}\left|h_{\vec{q}, \psi_{\epsilon}^{\epsilon}}(x, t)-w_{\epsilon}(x, t)\right|=\sup _{B_{1} \times[0, T]}\left|k_{\vec{q}}(x, t)-w_{\epsilon}^{\epsilon}(x, t)\right|,
$$

where $w_{\epsilon}^{\epsilon}(x, t)=w_{\epsilon}\left(\frac{x}{\epsilon}, \frac{t}{\epsilon^{2}}\right)$.

Now we would like to rescale the right hand side of inequality (50). Notice that by applying the function $F_{\vec{q}}$ to any function pair of continuous functions $w_{\epsilon}, \phi_{\epsilon}$ we obtain a continuous function $F_{\vec{q}}\left(w_{\epsilon}^{\epsilon}, \psi_{\epsilon}^{\epsilon}\right): B_{\frac{1}{\epsilon}} \times\left[0, \frac{T}{\epsilon^{2}}\right] \rightarrow \mathbb{R}^{2}$, which satisfies (via Duhamel's formula) the following eqaution:

$$
\begin{aligned}
\frac{d F_{\vec{q}}\left(w_{\epsilon}, \psi_{\epsilon}\right)}{d t}-\Delta F_{\vec{q}}\left(w_{\epsilon}, \psi_{\epsilon}\right)+\frac{\nabla_{v} W\left(w_{\epsilon}+V_{\vec{q}}\right)}{2} & =\Delta V_{\vec{q}} \text { in } B_{\frac{1}{\epsilon}} \times\left[0, \frac{T}{\epsilon^{2}}\right] \\
F_{\vec{q}}\left(w_{\epsilon}, \psi_{\epsilon}\right)(x, t) & =0 \text { on } \partial B_{\frac{1}{\epsilon}} \\
F_{\vec{q}}\left(w_{\epsilon}, \psi_{\epsilon}\right)(x, 0) & =\psi_{\epsilon}(x) .
\end{aligned}
$$

Let us define the function $\mathcal{L}_{\vec{q}}: \mathcal{C}_{B_{1} \times[0, T]} \times \mathcal{C}_{B_{1}} \rightarrow \mathcal{C}_{B_{1} \times[0, T]}$ as $\mathcal{L}_{\vec{q}}\left(w_{\epsilon}^{\epsilon}, \psi_{\epsilon}^{\epsilon}\right)(x, t)=F_{\vec{q}}\left(w_{\epsilon}, \psi_{\epsilon}\right)\left(\frac{x}{\epsilon}, \frac{y}{\epsilon^{2}}\right)$, where as before $w_{\epsilon}^{\epsilon}(x, t)=w_{\epsilon}\left(\frac{x}{\epsilon}, \frac{t}{\epsilon^{2}}\right)$ and similarly $\psi_{\epsilon}^{\epsilon}(x, t)=\psi_{\epsilon}\left(\frac{x}{\epsilon}, \frac{t}{\epsilon^{2}}\right)$. A simple computation shows that for any $w_{\epsilon}^{\epsilon}, \psi_{\epsilon}^{\epsilon}$ the function obtained by evaluating $\mathcal{L}_{\vec{q}}$ at $\left(w_{\epsilon}^{\epsilon}, \psi_{\epsilon}^{\epsilon}\right)$, denoted by $\mathcal{L}_{\vec{q}}\left(w_{\epsilon}^{\epsilon}, \psi_{\epsilon}^{\epsilon}\right)$, satisfies

$$
\begin{aligned}
\frac{d \mathcal{L}_{\vec{q}}\left(w_{\epsilon}^{\epsilon}, \psi_{\epsilon}^{\epsilon}\right)}{d t}-\Delta \mathcal{L}_{\vec{q}}\left(w_{\epsilon}^{\epsilon}, \psi_{\epsilon}^{\epsilon}\right)+\frac{\nabla_{v} W\left(w_{\epsilon}^{\epsilon}+U_{\vec{q}}\right)}{2 \epsilon^{2}} & =\Delta U_{\vec{q}} \text { in } B_{1} \times[0, T] \\
\mathcal{L}_{\vec{q}}\left(w_{\epsilon}^{\epsilon}, \psi_{\epsilon}^{\epsilon}\right)(x, t) & =0 \text { on } \partial B_{1} \\
\mathcal{L}_{\vec{q}}\left(w_{\epsilon}^{\epsilon}, \psi_{\epsilon}^{\epsilon}\right)(x, 0) & =\psi_{\epsilon}^{\epsilon}(x) .
\end{aligned}
$$


Using again Duhamel's formula we conclude that

$$
\begin{aligned}
\mathcal{L}_{\vec{q}}\left(w_{\epsilon}^{\epsilon}, \psi_{\epsilon}^{\epsilon}\right)(x, t)=\int_{0}^{t} \int_{B_{1}} \mathcal{H}_{B_{1}}(x, y, t-s) & \left(-\frac{\nabla_{v} W\left(w_{\epsilon}^{\epsilon}+U_{\vec{q}}\right)(y, s)}{\epsilon^{2}}\right. \\
\left.+\Delta U_{\vec{q}}(y)\right) d y d s & +\int_{B_{1}} \mathcal{H}_{B_{1}}(x, y, t) \psi_{\epsilon}^{\epsilon}(y) d y .
\end{aligned}
$$

In particular, we have that $k_{\vec{q}, \psi}$ defined by (56) is a fixed point of $\mathcal{L}_{\vec{q}}(\cdot, \psi)$.

Hence, the right hand side of equation (50) reads

$$
\sup _{B_{\frac{1}{\epsilon}} \times\left[0, \frac{T}{\epsilon^{2}}\right]}\left|F_{\vec{q}}\left(w_{\epsilon}, \psi_{\epsilon}\right)-w_{\epsilon}\right|=\sup _{B_{1} \times[0, T]}\left|\mathcal{L}_{\vec{q}}\left(w_{\epsilon}^{\epsilon}, \psi_{\epsilon}\right)-w_{\epsilon}^{\epsilon}\right| .
$$

In this context we can re-formulate Theorem 4.2 (dropping the super-indeces to simplify the notation) as

Theorem 4.3. Let $k_{\vec{q}, \psi}$ be defined by (56)). Then is the unique fixed point of $\mathcal{L}_{\vec{q}}(\cdot, \psi)$. Moreover, for any fixed $K>0$ and sequences of continuous functions $\psi_{n}, w_{n}$ satisfying $\sup \left|\psi_{n}\right|, \sup \left|w_{n}\right| \leq K$ and vectors $\vec{q}_{n} \in \mathcal{Q}$ and $T_{n}>0$ holds either

(1) $\sup _{B_{1} \times[0, T]}\left|k_{\vec{q}, \psi_{\epsilon}}(x, t)-w_{\epsilon}(x, t)\right| \rightarrow 0$ or

(2) there is a constant $C$, independent of $\epsilon, \sigma$ and $T$ such that

$$
\sup _{B_{1} \times[0, T]}\left|k_{\vec{q}, \psi_{\epsilon}}(x, t)-w_{\epsilon}(x, t)\right| \leq C \sup _{B_{1} \times[0, T]}\left|\mathcal{L}_{\vec{q}}\left(w_{\epsilon}, \psi_{\epsilon}\right)-w_{\epsilon}\right|,
$$

where $\vec{q}=(\epsilon, \sigma, \alpha)$.

Now we can devote ourselves to prove Theorem 4.1. We divide the proof into two steps: Lemma 4.2 and Lemma 4.3 .

Notice first that the function $k_{\vec{q}, \psi}$ defined by (56) is a solution to the following equation:

$$
\begin{aligned}
P k_{\vec{q}, \psi}+\frac{\nabla_{v} W\left(k_{\vec{q}, \psi}+U_{\vec{q}}\right)}{2 \epsilon^{2}} & =\Delta U_{\vec{q}} \text { in } B_{1} \\
k_{\vec{q}, \psi}(x, t) & =0 \text { on } \partial B_{1} \\
k_{\vec{q}, \psi}(x, 0) & =\psi .
\end{aligned}
$$

where $P k_{\vec{q}, \psi}=\frac{d k_{\vec{q}, \psi}}{d t}-\Delta k_{\vec{q}, \psi}$. In order to simplify the notation, when $\psi \equiv 0$ we will simply denote this solution by $k_{\vec{q}}$ (instead of $k_{\vec{q}, 0}$ ). In Lemma 4.2 we show that

$$
\lim _{\epsilon \rightarrow 0} \sup _{B_{1} \times[0, \infty]}\left|k_{\vec{q}}(x, t)\right|=0 .
$$

In order to do this computation we will use several estimates from the Appendix. Thereafter we will conclude the proof of Theorem 4.1 by showing in Lemma 4.3 that for every fixed $\epsilon$ there is a sequence $0<t_{n} \nearrow \infty$ satisfying

$$
\lim _{n \rightarrow \infty} \sup _{B_{1}}\left|k_{\vec{q}}\left(x, t_{n}\right)-u_{\epsilon}+U_{\vec{q}}\right|=0 .
$$

Lemma 4.2. Let $k_{\vec{q}}$ be the solution to (58)-(59)-(60), for $\psi=0$. Then

$$
\lim _{\epsilon \rightarrow 0} \sup _{B_{1} \times[0, \infty]}\left|k_{\vec{q}}(x, t)\right|=0 .
$$


Proof. Suppose that

$$
\sup _{B_{1} \times[0, \infty)}\left|k_{\vec{q}}\right| \not \nrightarrow 0 .
$$

Theorem 4.3 implies that (by choosing $w_{\epsilon}=\psi_{\epsilon}=0$ )

$$
\sup _{B_{1} \times[0, \infty)}\left|k_{\vec{q}}\right| \leq C \sup _{B_{1} \times[0, \infty)}\left|\mathcal{L}_{\vec{q}}(0,0)\right| .
$$

Set $S_{\epsilon}=\sup _{B_{1} \times[0, \infty)}\left|\mathcal{L}_{\vec{q}}(0,0)\right|$ (possibly infinity). Fix $\delta>0$ and notice that, by definition of supremum, there is a $t_{\epsilon}$ such that $\sup _{x \in B_{1}}\left|\mathcal{L}_{\vec{q}}(0,0)\left(x, t_{\epsilon}\right)-S_{\epsilon}\right| \leq \delta$ (or when $S_{\epsilon}=\infty$ pick $t_{\epsilon}$ such that $\left.\sup _{x \in B_{1}}\left|\mathcal{L}_{\vec{q}}(0,0)\left(x, t_{\epsilon}\right)\right| \geq \delta^{-1}\right)$.

We will show that, independently of $\delta$, holds $\sup _{x \in B_{1}}\left|\mathcal{L}_{\vec{q}}(0,0)\right|\left(x, t_{\epsilon}\right) \rightarrow 0$ as $\epsilon \rightarrow 0$ (notice that this immediately contradicts $\left.S_{\epsilon}=\infty\right)$. Recall first that

$$
\mathcal{L}_{\vec{q}}(0,0)(x, t)=\int_{0}^{t} \int_{B_{1}} \mathcal{H}_{B_{1}}(x, y, t-s)\left(-\frac{\nabla_{v} W\left(U_{\vec{q}}\right)(y, s)}{\epsilon^{2}}+\Delta U_{\vec{q}}(y)\right) d y d s .
$$

Notice that for $|x| \leq \epsilon^{\alpha}-E$ we have

$$
\frac{-\nabla_{v} W\left(U_{\vec{q}}\right)}{\epsilon^{2}}+\Delta U_{\vec{q}}=\frac{-\nabla_{v} W\left(u_{\sigma}^{\epsilon}\right)}{\epsilon^{2}}+\Delta u_{\sigma}^{\epsilon}=0 .
$$

Hence, (62) implies

$$
\left|\mathcal{L}_{\vec{q}}(0,0)\right|(x, t) \leq I_{1}(x, t)+I_{2}(x, t),
$$

where

$$
\begin{array}{r}
I_{1}(x, t)=\int_{0}^{t} \int_{\left\{|y| \geq \epsilon^{\alpha}\right\}} \mathcal{H}_{B_{1}}(x, y, t-s)\left|\frac{-\nabla_{v} W\left(\phi_{\epsilon}\right)}{\epsilon^{2}}+\Delta \phi_{\epsilon}\right|(y, s) d y d s \\
I_{2}(x, t)=\int_{0}^{t} \int_{\left\{\epsilon^{\alpha}-E \leq|y| \leq \epsilon^{\alpha}\right\}} \mathcal{H}_{B_{1}}(x, y, t-s) \mid \frac{-\nabla_{v} W\left(U_{\vec{q}}\right)}{\epsilon^{2}}+\eta_{\alpha}^{\epsilon} \Delta \phi_{\epsilon} \\
\quad+\Delta\left(\eta_{\alpha}^{\epsilon}\right)\left(h_{\sigma}^{\epsilon}-\phi_{\epsilon}\right)+\nabla\left(\eta_{\alpha}^{\epsilon}\right) \cdot D\left(u_{\sigma}^{\epsilon}-\phi_{\epsilon}\right) \mid(y, s) d y d s .
\end{array}
$$

Now we find bounds for $I_{1}$ and $I_{2}$. For each of these integrals we will consider two ranges for the variable $t$, namely $t \leq T$ and $t \geq T$, where $T>0$ is fixed positive constant.

- Bounds over $I_{1}$ :

Since $\epsilon<\epsilon^{\alpha}$ (when $\epsilon<1$ ) we have that for every $|x| \geq \epsilon^{\alpha}$ the function $\eta(x) \equiv 0$ and for such $x$ we have

$$
\begin{aligned}
& \Delta \phi_{\epsilon}(x)=\frac{1}{\epsilon^{2}}\left(\epsilon^{2} \Delta \eta_{6} \zeta_{31}\left(d_{0}\left(\frac{x}{\epsilon}\right)\right)+\eta_{6} \zeta_{31}^{\prime \prime}\left(d_{0}\left(\frac{x}{\epsilon}\right)\right)\right. \\
&+2 \epsilon \nabla \eta_{6} \cdot \nabla d_{0}\left(\frac{x}{\epsilon}\right) \zeta_{6}^{\prime}\left(d_{0}\left(\frac{x}{\epsilon}\right)\right)+\epsilon^{2} \Delta \eta_{5} c_{i} \\
&+\sum_{i=1}^{3} \epsilon^{2} \Delta \eta_{2 i} \zeta_{i i+1}\left(d_{i}\left(\frac{x}{\epsilon}\right)\right)+\eta_{2 i} \zeta_{i i+1}^{\prime \prime}\left(d_{i}\left(\frac{x}{\epsilon}\right)\right) \\
&\left.+2 \epsilon \nabla \eta_{2 i} \cdot \nabla d_{i}\left(\frac{x}{\epsilon}\right) \zeta_{i i+1}^{\prime}\left(d_{i}\left(\frac{x}{\epsilon}\right)\right)+\epsilon^{2} \Delta \eta_{2 i-1} c_{i}\right)
\end{aligned}
$$


Since the functions $\eta_{j}$ depend only on the angle $\theta$ we have that

$$
\begin{aligned}
\Delta \eta_{j} & =\frac{\eta_{j}^{\prime \prime}}{r^{2}} \text { and } \\
\left|\nabla \eta_{j}\right| & \leq\left|\eta_{j}^{\prime}\right| .
\end{aligned}
$$

In particular for $|x| \geq \epsilon^{\alpha}$

$$
\begin{aligned}
& \left|\Delta \eta_{j}\right| \leq \frac{4\left|\eta_{j}^{\prime \prime}\right|}{\epsilon^{2 \alpha}} \text { and } \\
& \left|\nabla \eta_{j}\right| \leq\left|\eta_{j}^{\prime}\right| .
\end{aligned}
$$

Recall that for $\theta \in\left[\theta_{i}-\frac{\delta}{2}, \theta_{i}+\frac{\delta}{2}\right]$ we have $\eta_{2 i} \equiv 1$ and $\eta_{j} \equiv 0$ for every $j \neq 2 i$. Then

$$
\frac{\nabla_{v} W\left(\phi_{\epsilon}\right)}{\epsilon^{2}}+\Delta \phi_{\epsilon}=0 \text { for } \theta \in\left[\theta_{i}-\frac{\delta}{2}, \theta_{i}+\frac{\delta}{2}\right]
$$

Now we need to find bounds for $\theta \in\left[\theta_{i}+\frac{\delta}{2}, \theta_{i+1}-\delta\right]$. Notice first that

$$
\begin{gathered}
|\Delta \eta(\theta)|=\left|\frac{\eta^{\prime \prime}(\theta)}{r^{2}}\right| \leq \frac{K}{r^{2}} \leq \frac{K}{\epsilon^{2 \alpha}} \text { for }|x| \geq \epsilon^{\alpha} \\
|\nabla \eta|=\left|\frac{\eta^{\prime}}{r}\right| \leq \frac{K}{r} \leq \frac{K}{\epsilon^{\alpha}} \text { for }|x| \geq \epsilon^{\alpha} .
\end{gathered}
$$

Notice also that $\eta_{j} \neq 0$ only for $j=21,2 i-1$ and

$$
\eta_{2 i}+\eta_{2 i-1}=1 \text {. }
$$

Hence

$$
\begin{aligned}
\Delta \phi_{\epsilon}=\frac{1}{\epsilon^{2}} & \left(\epsilon^{2} \Delta \eta_{2 i}(\theta) \zeta_{i i+1}\left(d_{i}\left(\frac{x}{\epsilon}\right)\right)+\eta_{2 i}(\theta) \zeta_{i i+1}^{\prime \prime}\left(d_{i}\left(\frac{x}{\epsilon}\right)\right)\right. \\
& \left.+2 \epsilon \nabla \eta_{2 i}(\theta) \cdot \nabla d_{i}\left(\frac{x}{\epsilon}\right) \zeta_{i i+1}^{\prime}\left(d_{i}\left(\frac{x}{\epsilon}\right)\right)+\epsilon^{2} \Delta \eta_{2 i-1}(\theta) c_{i}\right) \\
=\Delta & \eta_{2 i}(\theta)\left(\zeta_{i i+1}\left(d_{i}\left(\frac{x}{\epsilon}\right)\right)-c_{i}\right)+\eta_{2 i}(\theta) \frac{-\nabla_{v} W\left(\zeta_{i i+1}\right)}{\epsilon^{2}}\left(d_{i}\left(\frac{x}{\epsilon}\right)\right) \\
& +2 \frac{1}{\epsilon} \nabla \eta_{2 i}(\theta) \cdot \nabla d_{i}\left(\frac{x}{\epsilon}\right) \zeta_{i i+1}^{\prime}\left(d_{i}\left(\frac{x}{\epsilon}\right)\right) .
\end{aligned}
$$

Using Hypothesis 1 we have that there are constants $K, c>0$ such that

$$
\left|\frac{\nabla_{v} W\left(\phi_{\epsilon}\right)}{\epsilon^{2}}+\Delta \phi_{\epsilon}\right| \leq K \frac{e^{-c \frac{d_{i}}{\epsilon}}}{\epsilon^{2}} \text { for }|x| \geq \epsilon^{\alpha} \text { and } \theta \in\left[\theta_{i}+\frac{\delta}{2}, \theta_{i+1}-\frac{\delta}{2}\right] .
$$

Furthermore, for $|x|>\epsilon^{\alpha}$ and $\theta \in\left[\theta_{i}+\frac{\delta}{2}, \theta_{i+1}-\frac{\delta}{2}\right]$ we have

$$
\left|d_{i}\right| \geq \epsilon^{\alpha} \sin \delta
$$

Hence,

$$
\left|\frac{\nabla_{v} W\left(\phi_{\epsilon}\right)}{\epsilon^{2}}+\Delta \phi_{\epsilon}\right| \leq K \frac{e^{-c \frac{\epsilon^{\alpha} \sin \delta}{\epsilon}}}{\epsilon^{2}} \text { for }|x|>\epsilon^{\alpha} \text { and } \theta \in\left[\theta_{i}+\frac{\delta}{2}, \theta_{i+1}-\frac{\delta}{2}\right] .
$$

Now we proceed to find bounds in two different cases: 
(1) Suppose that $t \leq T$. Equations (64) and (68) imply

$$
I_{1}(x, t) \leq K \frac{e^{-c \frac{\epsilon^{\alpha} \sin \delta}{\epsilon}}}{\epsilon^{2}} \int_{0}^{t} \int_{\left\{|x| \geq \epsilon^{\alpha}\right\}} \mathcal{H}_{B_{1}}(x, y, t-s) d y d s .
$$

Using Lemma A-1 we have

$$
I_{1}(x, t) \leq K \frac{e^{-c \frac{\epsilon^{\alpha} \sin \delta}{\epsilon}}}{\epsilon^{2}} \text { for every } x \in B_{1} \text { and } 0 \leq t \leq T .
$$

(2) Suppose that $t \geq T$

Let

$$
f_{\epsilon}=\left|\frac{\nabla_{v} W\left(\phi_{\epsilon}\right)}{\epsilon^{2}}+\Delta \phi_{\epsilon}\right|
$$

and fix $\delta>0$. Now we divide $I_{1}$ in the three following integrals:

$$
\begin{aligned}
& I_{11}(x, t)=\int_{0}^{t-\delta} \int_{\left\{|y| \geq \epsilon^{\alpha}\right\} \cap\left\{|x-y| \leq \frac{\sqrt{t-s}}{t}\right\}} \mathcal{H}_{B_{1}}(x, y, t-s) f_{\epsilon}(y, s) d y d s, \\
& I_{12}(x, t)=\int_{0}^{t-\delta} \int_{\left\{|y| \geq \epsilon^{\alpha}\right\} \cap\left\{|x-y| \geq \frac{\sqrt{t-s}}{t}\right\}} \mathcal{H}_{B_{1}}(x, y, t-s) f_{\epsilon}(y, s) d y d s \\
& I_{13}(x, t)=\int_{t-\delta}^{\delta} \int_{\left\{|y| \geq \epsilon^{\alpha}\right\}} \mathcal{H}_{B_{1}}(x, y, t-s) f_{\epsilon}(y, s) d y d s .
\end{aligned}
$$

Then

$$
I_{1}=I_{11}+I_{12}+I_{13}
$$

By Theorem $\mathrm{A}-1$ we have that $\left|\mathcal{H}_{B_{1}}(x, y, t-s)\right| \leq \frac{C}{(t-s)}$, then

$$
\begin{aligned}
I_{11}(x, t) & \leq C \int_{0}^{t-\delta} \frac{\sup _{|y| \geq \alpha} f_{\epsilon}}{(t-s)} \frac{(t-s)}{t^{2}} \pi d s \\
& =\frac{\sup _{|y| \geq \alpha} f_{\epsilon}}{t^{2}}(t-\delta) \\
& \leq C \frac{e^{-c \frac{d_{i}}{\epsilon}}}{t \epsilon^{2}} .
\end{aligned}
$$

Using again Theorem A-1, for $|x-y| \geq \frac{\sqrt{t-s}}{t}$ we have $\left|\mathcal{H}_{B_{1}}(x, y, t-s)\right|=$ $O\left(\left[\frac{1}{t}\right]^{-\infty}\right)$. In particular there is a constant $C$ such that $\left|\mathcal{H}_{B_{1}}(x, y, t-s)\right| \leq \frac{C}{t}$, then

$$
\begin{aligned}
I_{12}(x, t) & \leq \int_{0}^{t-\delta} \frac{C}{t} \int_{B_{1}} f_{\epsilon}(y) d y \\
& \leq t \frac{C}{t} \int_{B_{1}} f_{\epsilon}(y) d y \\
& \leq C \frac{e^{-c \frac{d_{i}}{\epsilon}}}{\epsilon^{2}}
\end{aligned}
$$


Finally, using Lemma A-1 we have

$$
I_{13}(x, t) \leq \delta \sup f_{\epsilon} \leq C \frac{e^{-c \frac{d_{i}}{\epsilon}}}{\epsilon^{2}} .
$$

Combining the previous estimates we obtain

$$
I_{1}(x, t) \leq C \frac{e^{-c \frac{d_{i}}{\epsilon}}}{\epsilon^{2}} \text { for every } x \in B_{1} \text { and } t \geq T .
$$

- Bounds over $I_{2}$ :

Using the definitions of $U_{\vec{q}}, \phi_{\epsilon}$, Theorem A-2 and Lemma 2.2 we have

$$
\begin{gathered}
\left|\frac{-\nabla_{v} W\left(U_{\vec{q}}\right)}{\epsilon^{2}}+\eta_{\alpha}^{\epsilon} \Delta \phi_{\epsilon}\right| \leq \frac{C}{\epsilon^{2}} \\
\left|\Delta\left(\eta_{\alpha}^{\epsilon}\right)\left(h_{\sigma}^{\epsilon}-\phi_{\epsilon}\right)\right| \leq \frac{C}{E^{2}} \\
\left|\nabla\left(\eta_{\alpha}^{\epsilon}\right) \cdot D\left(u_{\sigma}^{\epsilon}-\phi_{\epsilon}\right)\right| \leq \frac{C}{E \epsilon} .
\end{gathered}
$$

Hence:

(1) For $t \leq T$

$$
I_{2}(x, t) \leq C \int_{0}^{t} \int_{\epsilon^{\alpha}-E \leq|x| \leq \epsilon^{\alpha}} \mathcal{H}(x, y, t-s)\left(\frac{1}{\epsilon^{2}}+\frac{1}{E^{2}}+\frac{1}{E \epsilon}\right) d y d s .
$$

Theorem A-1 implies that for $t-s \geq \epsilon^{m+2}$ there is a constant $C$ independent of $x, y$ such that $|\mathcal{H}(x, y, t-s)| \leq \frac{C}{\epsilon^{m+2}}$. Moreover, by definition $\epsilon^{\alpha} \leq E=$ $\epsilon^{\alpha}\left(2-\epsilon^{2 m+4}\right) \leq 2 \epsilon^{\alpha}$. Hence

$$
\begin{aligned}
I_{2} \leq & \int_{0}^{t-\epsilon^{m+2}} \int_{\epsilon^{\alpha}-E \leq|x| \leq \epsilon^{\alpha}} \frac{C}{\epsilon^{m+2}}\left(\frac{1}{\epsilon^{2}}+\frac{1}{\epsilon^{2 \alpha}}+\frac{1}{\epsilon^{1+\alpha}}\right) d y d s \\
& +\int_{t-\epsilon^{m+2}}^{t} \int_{\epsilon^{\alpha}-E \leq|x| \leq \epsilon^{\alpha}} \mathcal{H}_{B_{1}}(x, y, t-s) \frac{1}{\epsilon^{2}}\left(1+\epsilon^{2-2 \alpha}+\epsilon^{1-\alpha}\right) d y d s \\
\leq & \frac{C}{\epsilon^{m+4}} \int_{0}^{t-\epsilon^{m+2}}\left(1+\epsilon^{2-2 \alpha}+\epsilon^{1-\alpha}\right) \pi\left(\epsilon^{2 \alpha}-\left(\epsilon^{\alpha}-E\right)^{2}\right) d s \\
& +\frac{C}{\epsilon^{2}} \int_{t-\epsilon^{m+2}}^{t} \int_{B_{1}} \mathcal{H}(x, y, t-s) d y d s .
\end{aligned}
$$

Using that $t \leq T$, Lemma $A-1$ and the definition of $E$ we conclude

$$
\begin{aligned}
I_{2}(x, t) & \leq \frac{C}{\epsilon^{m+4}} E\left(2 \epsilon^{\alpha}-E\right)+\frac{C}{\epsilon^{2}} \epsilon^{m+2} \\
& \leq \frac{C}{\epsilon^{m+4}} \epsilon^{\alpha} \epsilon^{2 m+4-\alpha}+C \epsilon^{m} \\
& \leq C \epsilon^{m} \text { for } x \in B_{1} \text { and } 0 \leq t \leq T .
\end{aligned}
$$

(2) For $t \geq T$

The previous estimates show that the integrand of $I_{2}$ can be bounded by $\frac{C}{\epsilon^{2}}$. Dividing up the integral as we did for $I_{1}$ we obtain 


$$
\begin{aligned}
I_{2} \leq & \int_{0}^{t-\epsilon^{m+2}} \int_{\left\{\epsilon^{\alpha}-E \leq|y| \leq \epsilon^{\alpha}\right\} \cap\left\{|x-y| \leq \frac{\sqrt{t-s}}{t}\right\}} \mathcal{H}_{B_{1}}(x, y, t-s) \frac{C}{\epsilon^{2}} d y d s \\
& +\int_{0}^{t-\epsilon^{m+2}} \int_{\left\{\epsilon^{\alpha}-E \leq|y| \leq \epsilon^{\alpha}\right\} \cap\left\{|x-y| \geq \frac{\sqrt{t-s}}{t}\right\}} \mathcal{H}_{B_{1}}(x, y, t-s) \frac{C}{\epsilon^{2}} d y d s \\
& +\int_{t-\epsilon^{m+2}}^{t} \int_{\left\{\epsilon^{\alpha}-E \leq|y| \leq \epsilon^{\alpha}\right\}} \mathcal{H}_{B_{1}}(x, y, t-s) \frac{C}{\epsilon^{2}} d y d s .
\end{aligned}
$$

Using Hölder's inequality in the first integral for $p<2$ we get

$$
\begin{aligned}
I_{2} & \leq \int_{0}^{t-\epsilon^{m+2}}\left(\int_{\left\{\epsilon^{\alpha}-E \leq|y| \leq \epsilon^{\alpha}\right\}} \frac{C}{\epsilon^{2 p}} d y\right)^{\frac{1}{p}}\left(\int_{\left\{|x-y| \leq \frac{\sqrt{t-s}}{t}\right\}} \mathcal{H}_{B_{1}}^{q}(x, y, t-s) d y\right)^{\frac{1}{q}} d s \\
& +\int_{0}^{t-\epsilon^{m+2}} \int_{\left\{\epsilon^{\alpha}-E \leq|y| \leq \epsilon^{\alpha}\right\} \cap\left\{|x-y| \geq \frac{\sqrt{t-s}}{t}\right\}} \mathcal{H}_{B_{1}}(x, y, t-s) \frac{1}{\epsilon^{2}} d y d s+C \frac{\epsilon^{m+2}}{\epsilon^{2}} .
\end{aligned}
$$

As before, Theorem A-1 implies $\left|\mathcal{H}_{B_{1}}\right| \leq \frac{C}{t-s}$ and that for $|x-y| \geq \frac{t-s}{t}$ holds $\mathcal{H}_{B_{1}}(x, y, t-s)=O\left(\left(\frac{1}{t}\right)^{-\infty}\right)$, therefore

$$
\begin{aligned}
I_{2} \leq C & {\left[\int_{0}^{t-\epsilon^{m+2}}\left(\frac{C \epsilon^{2 m+4}}{\epsilon^{2 p}}\right)^{\frac{1}{p}} \frac{1}{t-s}\left(\frac{t-s}{t^{2}}\right)^{\frac{1}{q}} d s\right.} \\
& \left.+\int_{0}^{t-\epsilon^{m+2}} \int_{\left\{\epsilon^{\alpha}-E \leq|y| \leq \epsilon^{\alpha}\right\}} \frac{C}{t} \frac{\epsilon^{2 m+4}}{\epsilon^{2}}+C \frac{\epsilon^{m+2}}{\epsilon^{2}}\right] \\
\leq C & \left(\epsilon^{2 m+4-2 p}\right)^{\frac{1}{p}} \frac{t^{\frac{1}{q}}-\epsilon^{\frac{m+2}{q}}}{t^{\frac{2}{q}}}+t \frac{C}{t} \epsilon^{2 m+2}+C \epsilon^{m} .
\end{aligned}
$$

Therefore, for $t \geq T$ and $p<2$ holds

$$
I_{2}(x, t) \leq C\left(\frac{\epsilon^{\frac{2 m+4-2 p}{p}}}{T^{\frac{1}{q}}}+\epsilon^{2}+\epsilon^{2}\right) \leq C \epsilon^{2}
$$

Now we can conclude the result of Lemma by combining (69), (701), (711) in (72) in (63) and (61). More precisely:

$$
\sup _{B_{1} \times[0, \infty)}\left|k_{\vec{q}}\right| \leq C \frac{e^{-c \frac{\epsilon^{\alpha} \sin \delta}{\epsilon}}}{\epsilon^{2}}+C \epsilon^{m} \leq C \epsilon^{m},
$$

where $C$ depends on $\alpha$ and $m$. This implies the desired Lemma.

To finish the proof of Theorem 4.1 we need the following Lemma

Lemma 4.3. Fix $\epsilon>0$ and let $k_{\vec{q}}$ be the solution (58)- (59) -(60). Then, there is a sequence of times $t_{n} \nearrow \infty$ such that

$$
\lim _{n \rightarrow \infty} \sup _{B_{1}}\left|k_{\vec{q}}\left(x, t_{n}\right)-u_{\epsilon}(x)+U_{\vec{q}}(x)\right|=0 .
$$


Proof. Corollary A-2 in the appendix shows that for every $t>0$ there is a constant $C$ such that $\left|D k_{\vec{q}}(x, t)\right| \leq \frac{C}{\epsilon}$. Similarly, by taking derivatives on the equation, we can find bounds over the second and third space derivatives (these bounds will depend on $\epsilon$ ). Since $\epsilon$ is fixed, using Arzela-Ascoli's Theorem we conclude for every sequence $t_{n} \nearrow \infty$ there is a subsequence $k_{\vec{q}}\left(x, t_{n}\right)$ that converges in $\mathcal{C}^{2}$. Let us denote this limit by $k_{\vec{q}}^{\infty}(x)$ and the convergent subsequence $\left\{t_{n}\right\}_{n \in \mathbb{N}}$ as well.

We will show that $k_{\vec{q}}^{\infty}(x)$ satisfies

$$
\begin{gathered}
\Delta k_{\vec{q}}^{\infty}(x)=\frac{\nabla_{v} W\left(k_{\vec{q}}^{\infty}+U_{\vec{q}}\right)}{\epsilon^{2}}-\Delta U_{\vec{q}} \text { for } x \in B_{1} \\
\left.k_{\vec{q}}^{\infty}\right|_{\partial B_{1}}=0 .
\end{gathered}
$$

First we need to show that for every $\tau>0$ the sequence $k_{\vec{q}}\left(x, t_{n}+\tau\right)$ also converges in $\mathcal{C}^{2}$ to $k_{\vec{q}}^{\infty}(x)$. Define

$$
\mathcal{J}(t)=\int_{B_{1}}\left(\frac{\left|\nabla k_{\vec{q}}\right|^{2}}{2}+\frac{W\left(k_{\vec{q}}+U_{\vec{q}}\right)}{\epsilon^{2}}-k_{\vec{q}} \cdot \Delta U_{\vec{q}}\right)(x, t) d x
$$

Using Theorem $\mathrm{A}-2$ and the definition of $U_{\vec{q}}$ it is easy to see that $\mathcal{J}(t)$ is bounded below for every $t$. Moreover, taking time derivative we have

$$
\begin{aligned}
\frac{d \mathcal{J}}{d t} & =\int_{B_{1}}\left(\nabla k_{\vec{q}} \cdot \nabla\left(k_{\vec{q}}\right)_{t}+\frac{\nabla W\left(k_{\vec{q}}+U_{\vec{q}}\right)}{\epsilon^{2}} \cdot\left(k_{\vec{q}}\right)_{t}-\Delta U_{\vec{q}} \cdot\left(k_{\vec{q}}\right)_{t}\right)(x, t) d x \\
& =\int_{B_{1}}\left[\left(-\Delta k_{\vec{q}}+\frac{\nabla W\left(k_{\vec{q}}+U_{\vec{q}}\right)}{\epsilon^{2}}-\Delta U_{\vec{q}}\right) \cdot\left(k_{\vec{q}}\right)_{t}\right](x, t) d x \\
& =-\int_{B_{1}}\left|\left(k_{\vec{q}}\right)_{t}\right|^{2}(x, t) d x .
\end{aligned}
$$

Therefore $\mathcal{J}$ is bounded below and decreasing, hence it converges. Moreover for every fixed $\tau>0$

$$
\int_{t_{n}}^{t_{n}+\tau} \int_{B_{1}}\left|k_{\vec{q}}\right|_{t}^{2}(x, s) d x d s=\mathcal{J}\left(t_{n}\right)-\mathcal{J}\left(t_{n}+\tau\right) \rightarrow 0
$$

Since for every fixed $x$ we can write $k_{\vec{q}}\left(x, t_{n}+\tau\right)-k_{\vec{q}}\left(x, t_{n}\right)=\int_{t_{n}}^{t_{n}+\tau}\left(k_{\vec{q}}\right)_{t}(x, s) d s$, we have that

$$
\begin{aligned}
\int_{B_{1}}\left|k_{\vec{q}}\left(x, t_{n}+\tau\right)-k_{\vec{q}}\left(x, t_{n}\right)\right| d x & \leq \int_{t_{n}}^{t_{n}+\tau} \int_{B_{1}}\left|\left(k_{\vec{q}}\right)_{t}\right|(x, s) d x d s \\
& \leq C\left(\int_{t_{n}}^{t_{n}+\tau} \int_{B_{1}}\left|\left(k_{\vec{q}}\right)_{t}\right|^{2}(x, s) d x d s\right)^{\frac{1}{2}} \rightarrow 0 \text { as } n \rightarrow \infty .
\end{aligned}
$$

Hence $k_{\vec{q}}\left(x, t_{n}+\tau\right)-k_{\vec{q}}\left(x, t_{n}\right)$ converges to 0 almost everywhere. Let us show that this convergence is also uniform. Suppose that $\sup _{x \in B_{1}}\left|k_{\vec{q}}\left(x, t_{n}+\tau\right)-k_{\vec{q}}\left(x, t_{n}\right)\right| \not \rightarrow 0$ as $n \rightarrow \infty$. Then there is a $\delta>0$ and a subsequence of times such that

$$
\sup _{x \in B_{1}}\left|k_{\vec{q}}\left(x, t_{n}+\tau\right)-k_{\vec{q}}\left(x, t_{n}\right)\right| \geq \delta .
$$

As before, there is subsequence of these $\left\{t_{n}\right\}$ that converges uniformly. Since it converges almost everywhere to 0 , the uniform limit must be 0 contradicting (75). 
Since $\mathcal{J}\left(t_{n}\right)-\mathcal{J}\left(t_{n}+\tau\right) \rightarrow 0$, from the definition for $\mathcal{J}$ and the previous estimate we can see that

$$
\int_{B_{1}}\left(\left|\nabla k_{\vec{q}}\right|^{2}\left(x, t_{n}\right)-\left|\nabla k_{\vec{q}}\right|^{2}\left(x, t_{n}+\tau\right)\right) d x \rightarrow 0 \text { as } n \rightarrow \infty .
$$

As above we can conclude that this convergence is almost everywhere and uniform. Standard parabolic estimates imply that also $k_{\vec{q}}\left(x, t_{n}+\tau\right)-k_{\vec{q}}\left(x, t_{n}\right)$ in the $\mathcal{C}^{2}$ norm.

Now we can prove that $k_{\vec{q}}^{\infty}$ is a solution to the elliptic equation (73). Since $k_{\vec{q}}$ solves equation (58)-(59)-(160), we have that for any $\varphi \in C^{\infty}\left(B_{1}\right)$

$$
\begin{gathered}
\int_{B_{1}}\left(k_{\vec{q}}\left(y, t_{n}+1\right)-k_{\vec{q}}\left(y, t_{n}\right)\right) \varphi(y) d y= \\
\int_{t_{n}}^{t_{n}+1} \int_{B_{1}}\left(\Delta k_{\vec{q}}\left(y, t_{n}+\tau\right)-\frac{\nabla_{v} W\left(k_{\vec{q}}^{\infty}\right)}{\epsilon^{2}}\left(y, t_{n}+\tau\right)-\Delta U_{\vec{q}}\right) \varphi(y) d y d \tau .
\end{gathered}
$$

Letting $n \rightarrow \infty$ we get

$$
\int_{B_{1}}\left(\Delta k_{\vec{q}}^{\infty}-\frac{\nabla_{v} W\left(k_{\vec{q}}^{\infty}\right)}{\epsilon^{2}}-\Delta U_{\vec{q}}\right) \varphi(y) d y=0 .
$$

Moreover, since for every $t$ holds $\left.k_{\vec{q}}(x, t)\right|_{\partial B_{1}}=0$ it must hold $\left.k_{\vec{q}}^{\infty}\right|_{\partial B_{1}}=0$. Uniqueness of solution implies that necessarily $k_{\vec{q}}^{\infty} \equiv u_{\epsilon}-U_{\vec{q}}$, which proves the Lemma.

Now the proof of Theorem 4.1 is direct

Proof of Theorem 4.1. Fix $\epsilon>0$ and $m>0$. Consider $t_{n}$ as in Lemma 4.3, then

$$
\begin{aligned}
\sup _{B_{1}}\left|u_{\epsilon}-U_{\vec{q}}\right| & \leq \sup _{B_{1}}\left|u_{\epsilon}(x)-U_{\vec{q}}(x)-k_{\vec{q}}\left(x, t_{n}\right)\right|+\sup _{B_{1} \times[0, \infty)}\left|k_{\vec{q}}(x, t)\right| \\
& \leq \sup _{B_{1}}\left|u_{\epsilon}(x)-U_{\vec{q}}(x)-k_{\vec{q}}\left(x, t_{n}\right)\right|+C \epsilon^{m} .
\end{aligned}
$$

Taking $t_{n} \rightarrow \infty$ we have

$$
\sup _{B_{1}}\left|u_{\epsilon}-U_{\vec{q}}\right| \leq C \epsilon^{m} .
$$

Recalling the definition of $U_{\vec{q}}$ we have the result.

It is easy to see that the size of the radius of the inner ball in Theorem 4.1 (that is the ball where $u_{\epsilon}(x)-u_{\sigma}\left(\frac{\sigma x}{\epsilon}\right)$ converges to 0$)$ can be increased to $\epsilon^{\alpha}$. Namely, we let

$$
\tilde{U}_{\vec{q}}(y)=\tilde{\eta}_{\alpha}^{\epsilon}(y) \phi_{\epsilon}(y)+\left(1-\tilde{\eta}_{\alpha}^{\epsilon}(y)\right) u_{\sigma}(y),
$$

where $\tilde{\eta}: \mathbb{R} \rightarrow \mathbb{R}$ is a positive function such that $\tilde{\eta}(x)=0$ for $|x| \leq 1$ and $\tilde{\eta}(x)=2$ for $|x| \geq 1$ and

$$
\tilde{\eta}_{\alpha}^{\epsilon}(y)=\tilde{\eta}\left(\frac{1}{2 \tilde{E}}|y|+2-\frac{2 \epsilon^{\alpha}}{2 \tilde{E}}\right)
$$

with $\tilde{E}=4 \epsilon^{\alpha}-\epsilon^{2 m+4-\alpha}$. As before, $\alpha>0$.

Notice that

$$
\tilde{U}_{\vec{q}}(x)=\left\{\begin{array}{cl}
\phi_{\epsilon}(x) & \text { for }|x| \geq 2 \epsilon^{\alpha} \\
u_{\sigma}\left(\frac{\sigma x}{\epsilon}\right) & \text { for }|x| \leq 2 \epsilon^{\alpha}-E
\end{array} .\right.
$$

Hence, following the proof of Theorem 4.1, but changing $U_{\vec{q}}$ for $\tilde{U}_{\vec{q}}$ we have 
Corollary 4.2. Fix $0<\alpha<1$. Let $0<\sigma \leq 2 \epsilon^{1-\alpha}$. Then for every $m>0$ there is a constant $C$ (that might depend on $\alpha$ and $m$ ) such that

$$
\begin{gathered}
\sup _{|x| \geq 2 \epsilon^{\alpha}}\left|u_{\epsilon}-\phi_{\epsilon}\right| \leq C \epsilon^{m} . \\
\sup _{|x| \leq \epsilon^{\alpha}}\left|u_{\epsilon}(x)-u_{\sigma}\left(\frac{\sigma x}{\epsilon}\right)\right| \leq C \epsilon^{m} .
\end{gathered}
$$

Using Lemma 2.2 and 2.3 we can also prove

Corollary 4.3. Fix $0<\alpha<1$. Let $0<\sigma \leq 2 \epsilon^{1-\alpha}$. Then for every $m>0$ there is a constant $C$ (that might depend on $\alpha$ and $m$ ) such that

$$
\begin{gathered}
\sup _{|x| \geq \epsilon^{\alpha}}\left|D u_{\epsilon}-D \phi_{\epsilon}\right| \leq C \epsilon^{m} . \\
\sup _{|x| \leq \epsilon^{\alpha}}\left|D u_{\epsilon}(x)-\frac{\sigma}{\epsilon} D u_{\sigma}\left(\frac{\sigma x}{\epsilon}\right)\right| \leq C \epsilon^{m} .
\end{gathered}
$$

Proof. We start by proving the first inequality of the corollary. To prove this inequality we estimate separately in two different sets, namely we first prove the inequality for $x \in$ $B_{1-\frac{\epsilon^{\alpha}}{2}} \backslash B_{\epsilon^{\alpha}}$ and then for $x \in B_{1} \backslash B_{1-\frac{\epsilon^{\alpha}}{2}}$ (in fact, in the second step we find a bound in a larger set: $\left.B_{1} \backslash B_{\frac{3}{4}}\right)$.

We consider the function $u_{\epsilon}-\phi_{\epsilon}$ in the domain $B_{1} \backslash B_{\frac{\epsilon}{2}}$. Then

$$
\Delta\left(u_{\epsilon}-\phi_{\epsilon}\right)=\frac{\nabla W\left(u_{\epsilon}\right)-\nabla W\left(\phi_{\epsilon}\right)}{\epsilon^{2}}-\Delta \phi_{\epsilon}+\frac{\nabla W\left(\phi_{\epsilon}\right)}{\epsilon^{2}} .
$$

Using Lemma 2.2 we have for every $x \in B_{1-\frac{\epsilon^{\alpha}}{2}} \backslash B_{\epsilon^{\alpha}}$

$$
\begin{aligned}
&\left|D\left(u_{\epsilon}-\phi_{\epsilon}\right)\right|^{2}(x) \leq C\left(\sup _{|x| \geq \frac{\epsilon^{\alpha}}{2}}\left|u_{\epsilon}-\phi_{\epsilon}\right| \sup _{|x| \geq \frac{\epsilon^{\alpha}}{2}}\left|\frac{\nabla W\left(u_{\epsilon}\right)-\nabla W\left(\phi_{\epsilon}\right)}{\epsilon^{2}}-\Delta \phi_{\epsilon}+\frac{\nabla W\left(\phi_{\epsilon}\right)}{\epsilon^{2}}\right|\right. \\
&\left.+\frac{1}{\epsilon^{\alpha}} \sup _{|x| \geq \frac{\epsilon^{\alpha}}{2}}\left|u_{\epsilon}-\phi_{\epsilon}\right|^{2}\right) \\
& \leq C\left(\frac{M}{\epsilon^{2}} \sup _{|x| \geq \frac{\epsilon^{\alpha}}{2}}\left|u_{\epsilon}-\phi_{\epsilon}\right|^{2}+\left|u_{\epsilon}-\phi_{\epsilon}\right| \sup _{|x| \geq \frac{\epsilon^{\alpha}}{2}}\left|-\Delta \phi_{\epsilon}+\frac{\nabla W\left(\phi_{\epsilon}\right)}{\epsilon^{2}}\right|\right. \\
&\left.+\frac{1}{\epsilon^{\alpha}} \sup _{|x| \geq \frac{\epsilon^{\alpha}}{2}}\left|u_{\epsilon}-\phi_{\epsilon}\right|^{2}\right) .
\end{aligned}
$$

Using Theorem 4.1 and the estimates for $\left|-\Delta \phi_{\epsilon}+\frac{\nabla W\left(\phi_{\epsilon}\right)}{\epsilon^{2}}\right|$ in its proof we have for $m>0$ a constant $C$ (that depends on $m$ and $\alpha$ ) such that

$$
\left|D\left(u_{\epsilon}-\phi_{\epsilon}\right)\right|^{2}(x) \leq C \epsilon^{m},
$$

for $x \in B_{1-\frac{\epsilon^{\alpha}}{2}} \backslash B_{\epsilon^{\alpha}}$. 
In order to find bounds for $x \in B_{1} \backslash B_{1-\frac{\epsilon^{\alpha}}{2}}$ we consider a smooth function $\eta$ such that $\eta(x) \equiv 1$ for $x \geq \frac{3}{4}$ and $\eta \equiv 0$ for $x \leq \frac{1}{2}$ and we consider the function $\eta\left(u_{\epsilon}-\phi\right)$ (notice that in fact this will provide bounds in a larger set, namely $\left.B_{1} \backslash B_{\frac{3}{4}}\right)$. Then $\eta\left(u_{\epsilon}-\phi\right)$ satisfies $\Delta\left(\eta\left(u_{\epsilon}-\phi\right)\right)=\Delta \eta\left(u_{\epsilon}-\phi\right)+\nabla \eta \nabla\left(u_{\epsilon}-\phi\right)+\eta\left(\frac{\nabla W\left(u_{\epsilon}\right)-\nabla W\left(\phi_{\epsilon}\right)}{\epsilon^{2}}-\Delta \phi_{\epsilon}+\frac{\nabla W\left(\phi_{\epsilon}\right)}{\epsilon^{2}}\right)$.

Lemma 2.3, Theorem 4.1 and the previous estimates imply that

$$
\left|D\left(\eta\left(u_{\epsilon}-\phi\right)\right)\right|^{2}(x)=\left|D\left(u_{\epsilon}-\phi\right)\right|^{2}(x) \leq C \epsilon^{m} \text { for } \frac{3}{4} \leq|x| \leq 1,
$$

finishing the proof of the first inequality.

Now we need to prove the second inequality. Let $u_{\sigma}^{\epsilon}(x)=u_{\sigma}\left(\frac{\sigma x}{\epsilon}\right)$. To prove the second estimate we consider $u_{\epsilon}(x)-u_{\sigma}^{\epsilon}(x)$ in $B_{\frac{3 \epsilon}{2} \alpha}$. Since

$$
\Delta\left(u_{\epsilon}-u_{\sigma}^{\epsilon}\right)=\frac{\nabla W\left(u_{\epsilon}\right)-\nabla W\left(u_{\sigma}^{\epsilon}\right)}{\epsilon^{2}},
$$

Lemma 2.2 implies for every $x \in B_{\epsilon^{\alpha}}$

$$
\begin{gathered}
\left|D\left(u_{\epsilon}-u_{\sigma}^{\epsilon}\right)\right|^{2} \leq C\left(\sup _{|x| \leq \frac{\epsilon^{\alpha}}{2}}\left|u_{\epsilon}-u_{\sigma}^{\epsilon}\right| \sup _{|x| \leq \frac{\epsilon^{\alpha}}{2}}\left|\frac{\nabla W\left(u_{\epsilon}\right)-\nabla W\left(u_{\sigma}^{\epsilon}\right)}{\epsilon^{2}}\right|\right. \\
\left.\quad+\frac{1}{\epsilon^{\alpha}} \sup _{|x| \leq \frac{\epsilon^{\alpha}}{2}}\left|u_{\epsilon}-u_{\sigma}^{\epsilon}\right|^{2}\right) \\
\leq C\left(\frac{1}{\epsilon^{2}}+\frac{1}{\epsilon^{\alpha}}\right) \sup _{|x| \leq \frac{\epsilon^{\alpha}}{2}}\left|u_{\epsilon}-u_{\sigma}^{\epsilon}\right|^{2}
\end{gathered}
$$

Corollary 4.2 implies that for every $m>0$ there is a constant $C$ such that

$$
\left|D\left(u_{\epsilon}-u_{\sigma}^{\epsilon}\right)\right|^{2} \leq C \epsilon^{m}
$$

which finishes the proof.

\section{Proof of Theorem 1.1}

Let

$$
v_{\epsilon}(x)=u_{\epsilon}(\epsilon x)
$$

It holds

$$
\begin{aligned}
-\Delta v_{\epsilon}+\nabla_{v} W\left(v_{\epsilon}\right) & =0 \text { for } x \in B_{\frac{1}{\epsilon}}, \\
v_{\epsilon}(x) & =\phi(x) \text { for } x \in \partial B_{\frac{1}{\epsilon}} .
\end{aligned}
$$

We define the following sequence of continuous function $\tilde{v}_{\epsilon}: \mathbb{R}^{2} \rightarrow \mathbb{R}^{2}$

$$
\tilde{v}_{\epsilon}(x)=\left\{\begin{array}{cc}
v_{\epsilon}(x) & \text { for }|x| \leq \frac{1}{\epsilon} \\
\phi(x) & \text { if }|x| \geq \frac{1}{\epsilon}
\end{array}\right.
$$

We will divide the proof of Theorem 1.1 into two different theorems: Theorem 5.1 and Theorem 5.2. First we prove 
Theorem 5.1. There is a subsequence of $\tilde{v}_{\epsilon}$ such that $\tilde{v}_{\epsilon} \rightarrow v$ uniformly on compact sets as $\epsilon \rightarrow 0$ and $v$ satisfies

$$
\begin{gathered}
-\Delta v+\nabla_{v} W(v)=0 \text { for } x \in \mathbb{R}^{2}, \\
\lim _{|x| \rightarrow \infty}|v(x)-\phi(x)|=0 .
\end{gathered}
$$

Proof. Recall first that $\tilde{v}_{\epsilon}$ is given by (79). We will use the following strategy to prove Theorem 5.1:

(1) Using the results of Section 4, we show that $\tilde{v}_{\epsilon}$ is a Cauchy sequence in the sup norm. Therefore, $\tilde{v}_{\epsilon}$ has a uniform limit $v$.

(2) Using the definition of $\tilde{v}_{\epsilon}$ and the first step we show that the limit $v$ satisfies (81).

(3) Finally, we represent $v_{\epsilon}$ via Green's formula in compact sets. Taking limits, we conclude that $v$ satisfies (80).

Now we prove these steps:

Proof of Step $1:\left\{\tilde{v}_{\epsilon}\right\}$ is a Cauchy sequence in the sup norm.

Consider $\delta>0$ and take $0<\sigma<\epsilon<1$. We will show that there is an $\epsilon_{0}$ such that for every $0<\sigma<\epsilon<\epsilon_{0}$

$$
\left|\tilde{v}_{\epsilon}(x)-\tilde{v}_{\sigma}(x)\right| \leq \delta \text { for every } x \in \mathbb{R}^{2} .
$$

We will mainly use Theorem 4.1 and Corollary 4.2 with $\alpha=\frac{1}{2}$.

- If $|x| \leq \epsilon^{-\frac{1}{2}}$ :

Notice first, that also holds $|x| \leq \sigma^{-\frac{1}{2}}$ (since $\sigma<\epsilon$ ). By the definitions of $\tilde{v}_{\epsilon}$ and $v_{\epsilon}$ we have that

$$
\begin{aligned}
\tilde{v}_{\epsilon}(x)-\tilde{v}_{\sigma}(x) & =u_{\epsilon}(\epsilon x)-u_{\sigma}(\sigma x) \\
& =u_{\epsilon}(y)-u_{\sigma}\left(\frac{\sigma y}{\epsilon}\right),
\end{aligned}
$$

where $y=\epsilon x$. Notice that $|y|=\epsilon|x| \leq \epsilon^{\frac{1}{2}}$. Corollary 4.2 implies that there is a $\epsilon_{0}$ such that for every $\epsilon<\epsilon_{0}$

$$
\left|\tilde{v}_{\epsilon}(x)-\tilde{v}_{\sigma}(x)\right| \leq \delta \text { for }|x| \leq \epsilon^{-\frac{1}{2}}
$$

- If $|x| \geq \epsilon^{-\frac{1}{2}}$ and $|x| \geq \sigma^{-\frac{1}{2}}$ :

By the definition of $\phi$ and $\phi_{\epsilon}$ we have that

$$
\phi(x)=\phi_{\epsilon}(\epsilon x)=\phi_{\sigma}(\sigma x) .
$$

This implies

$$
\begin{array}{r}
\left|\tilde{v}_{\epsilon}(x)-\tilde{v}_{\sigma}(x)\right| \leq\left|\tilde{v}_{\epsilon}(x)-\phi_{\epsilon}(\epsilon x)\right|+\left|\phi_{\sigma}(\sigma x)-\tilde{v}_{\sigma}(x)\right| . \\
\text { If }|x| \geq \epsilon^{-1} \text {, by definition } \tilde{v}_{\epsilon}(x)=\phi_{\epsilon}(\epsilon x) \text {, hence } \\
\left|\tilde{v}_{\epsilon}(x)-\phi_{\epsilon}(\epsilon x)\right|=0 .
\end{array}
$$

For $\epsilon^{-\frac{1}{2}} \leq|x| \leq \epsilon^{-1}$, by definition $\tilde{v}_{\epsilon}(x)=u_{\epsilon}(\epsilon x)$. It also holds that $|\epsilon x| \geq \epsilon \epsilon^{-\frac{1}{2}}$. Therefore, Theorem 4.1 implies that there is an $\epsilon_{1}$ such that for every $\epsilon<\epsilon_{1}$

$$
\left|\tilde{v}_{\epsilon}(x)-\phi_{\epsilon}(\epsilon x)\right| \leq \frac{\delta}{2} \text { for } x \in B_{1} .
$$


Combining (84) and (85) we have that for $\epsilon<\epsilon_{1}$

$$
\left|\tilde{v}_{\epsilon}(x)-\phi_{\epsilon}(\epsilon x)\right| \leq \frac{\delta}{2} \text { for }|x| \geq \epsilon^{-\frac{1}{2}} .
$$

Since $\sigma<\epsilon<\epsilon_{1}$ it also holds that

$$
\left|\tilde{v}_{\sigma}(x)-\phi_{\sigma}(\sigma x)\right| \leq \frac{\delta}{2} \text { for }|x| \geq \sigma^{-\frac{1}{2}}
$$

Equations (83), (86) and (87) imply that

$$
\left|\tilde{v}_{\epsilon}(x)-\tilde{v}_{\sigma}(x)\right| \leq \delta \text { for }|x| \geq \epsilon^{-\frac{1}{2}},|x| \geq \sigma^{-\frac{1}{2}} .
$$

- If $\epsilon^{-\frac{1}{2}} \leq|x| \leq \sigma^{-\frac{1}{2}}$ :

Let us fix any $x$ in this range and define $\tilde{\sigma}=\frac{1}{|x|^{2}}$. As before,

$$
\phi(x)=\phi_{\epsilon}(\epsilon x)=\phi_{\tilde{\sigma}}(\tilde{\sigma} x) .
$$

Then, we have

$$
\begin{gathered}
\left|\tilde{v}_{\epsilon}(x)-\tilde{v}_{\sigma}(x)\right| \leq\left|\tilde{v}_{\epsilon}(x)-\phi_{\epsilon}(\epsilon x)\right|+\left|\phi_{\tilde{\sigma}}(\tilde{\sigma} x)-u_{\tilde{\sigma}}(\tilde{\sigma} x)\right| \\
+\left|u_{\tilde{\sigma}}(y)-u_{\sigma}\left(\frac{\sigma y}{\tilde{\sigma}}\right)\right|,
\end{gathered}
$$

where $y=\tilde{\sigma} x$. As before if $|x| \geq \epsilon^{-1}$, by definition

$$
\left|\tilde{v}_{\epsilon}(x)-\phi_{\epsilon}(\epsilon x)\right|=0 \text {. }
$$

If $\epsilon^{-\frac{1}{2}} \leq|x| \leq \epsilon^{-1}$, by definition $\tilde{v}_{\epsilon}(x)=u_{\epsilon}(\epsilon x)$. Hence, Theorem 4.1 implies that there is a $\epsilon_{2}$ such that for every $\epsilon<\epsilon_{2}$

$$
\left|\tilde{v}_{\epsilon}(x)-\phi_{\epsilon}(\epsilon x)\right| \leq \frac{\delta}{3} .
$$

Combining (90) and (91) we have for $\epsilon<\epsilon_{2}$

$$
\left|\tilde{v}_{\epsilon}(x)-\phi_{\epsilon}(\epsilon x)\right| \leq \frac{\delta}{3} \text { for } \epsilon^{-\frac{1}{2}} \leq|x| \leq \sigma^{-\frac{1}{2}}
$$

By the definition of $\tilde{\sigma}$ we have that $|\tilde{\sigma} x|=\frac{1}{|x|}=\tilde{\sigma}^{\frac{1}{2}}$ and $\tilde{\sigma} \leq \epsilon$. Hence, using Theorem 4.1 for $\tilde{\sigma} \leq \epsilon<\epsilon_{2}$ we have

$$
\left|\phi_{\tilde{\sigma}}(\tilde{\sigma} x)-u_{\tilde{\sigma}}(\tilde{\sigma} x)\right| \leq \frac{\delta}{3} \text { for } \epsilon^{-\frac{1}{2}} \leq|x| \leq \sigma^{-\frac{1}{2}} .
$$

Finally, as $|\tilde{\sigma} x|=\tilde{\sigma}^{\frac{1}{2}}$ and $\sigma \leq \tilde{\sigma} \leq \tilde{\sigma}^{\frac{1}{2}}$, Corollary 4.2 implies that there is a $\epsilon_{3}$ such that

$$
\left|u_{\tilde{\sigma}}(\tilde{\sigma} x)-\tilde{v}_{\sigma}\left(\frac{\tilde{\sigma} x}{\tilde{\sigma}}\right)\right| \leq \frac{\delta}{3} \text { for } \epsilon^{-\frac{1}{2}} \leq|x| \leq \sigma^{-\frac{1}{2}} .
$$

Equations (92), (93) and (94) in (89) imply that

$$
\left|\tilde{v}_{\epsilon}(x)-\tilde{v}_{\sigma}(x)\right| \leq \delta \text { for } \epsilon^{-\frac{1}{2}} \leq|x| \leq \sigma^{-\frac{1}{2}} .
$$


Combining equations (82), (88) and (95) we conclude that $\tilde{v}_{\epsilon}: \mathbb{R}^{2} \rightarrow \mathbb{R}^{2}$ is a Cauchy sequence in the sup norm, hence there is a continuous function $v(x)$ such that $\tilde{v}_{\epsilon} \rightarrow v$ uniformly in $\mathbb{R}^{2}$ as $\epsilon \rightarrow 0$.

Proof of Step 2; $v$ satisfies (81) Consider any sequence of points $x_{n}$ such that $\left|x_{n}\right| \rightarrow \infty$. Showing that $\lim _{n \rightarrow \infty}\left|v\left(x_{n}\right)-\phi\left(x_{n}\right)\right|=0$ is equivalent to (81). Let $\epsilon_{n}=\frac{1}{\mid x_{n}}$. Then for any $\beta>0$ the definition of $\tilde{v}_{\epsilon_{n}}$ implies:

$$
\begin{aligned}
\left|v\left(x_{n}\right)-\phi\left(x_{n}\right)\right| & =\left|v\left(x_{n}\right)-\tilde{v}_{\epsilon_{n}}\left(x_{n}\right)\right| \\
& \leq \sup _{\mathbb{R}^{2}}\left|v(x)-\tilde{v}_{\epsilon_{n}}(x)\right|
\end{aligned}
$$

Taking $n \rightarrow \infty$, step (11) implies that

$$
\lim _{n \rightarrow \infty}\left|v\left(x_{n}\right)-\phi\left(x_{n}\right)\right| \rightarrow 0,
$$

which finishes the proof.

\section{Proof of Step 3: $v$ satisfies (80)}

Let us fix a ball of radius $\rho$ in $\mathbb{R}^{2}$.

In every fixed ball $B_{\rho}$ we can use Green's formula to represent $v_{\epsilon}$. We have for $\epsilon \leq \frac{1}{\rho}$ that

$$
v_{\epsilon}(x)=-\int_{\partial B_{\rho}} v_{\epsilon}(y) \frac{\partial \mathcal{K}}{\partial \nu}(x, y) d S(y)+\int_{B_{\rho}} \nabla_{v} W\left(v_{\epsilon}\right)(y) \mathcal{K}(x, y) d y
$$

where $\mathcal{K}$ is the Green's function in the ball. Since in $B_{\rho}$ we have $v_{\epsilon} \rightarrow v$ uniformly as $\epsilon \rightarrow 0$, the function $v$ satisfies

$$
v(x)=-\int_{\partial B_{\rho}} v(y) \frac{\partial \mathcal{K}}{\partial \nu}(x, y) d S(y)+\int_{B_{\rho}} \nabla_{v} W(v)(y) \mathcal{K}(x, y) d y .
$$

Hence,

$$
-\Delta v+\nabla_{v} W(v)=0 \text { for } x \in B_{\rho} .
$$

Since this is true for arbitrary $x$ and $\rho$ we have that $v$ satisfies (80) for every $x \in \mathbb{R}^{2}$, which concludes the proof of the Theorem.

Now we finish the Proof of Theorem 1.1 by showing

Theorem 5.2. Let

$$
\mathcal{V}=\left\{w \in C^{1}: \int_{\mathbb{R}^{2}}|D w-D \phi| d x, \int_{\mathbb{R}^{2}}|w-\phi| d x<\infty\right\}
$$

Define the energy functional

$$
\mathcal{G}(w)=\left\{\begin{array}{cl}
\int_{\mathbb{R}^{2}}\left(|D w|^{2}+W(w)-|D \phi|^{2}-W(\phi)\right) d y & \text { if } w \in \mathcal{V} \\
\infty & \text { otherwise. }
\end{array}\right.
$$

The energy $\mathcal{G}$ is bounded below and the solution $v$ described by Theorem 5.1 minimizes $\mathcal{G}$. That is

$$
\mathcal{G}(v)=\inf _{w \in C^{1}} \mathcal{G}(w)
$$


Proof. Define

$$
\tilde{\mathcal{G}}_{\epsilon}(w)=\left\{\begin{array}{cl}
\int_{B_{\epsilon^{-1}}}|D w|^{2}+W(w) d y & \text { if } w \in H^{1}\left(B_{\epsilon^{-1}}\right) \text { and }\left.w\right|_{\partial B_{\epsilon^{-1}}}(x)=\phi_{\epsilon}(x) \\
\infty & \text { otherwise }
\end{array}\right.
$$

and consider $v_{\epsilon}$ as in the previous Theorem. We will divide the proof of Theorem 5.2 into the following steps:

(1) $v_{\epsilon}$ is a minimizer for $\tilde{\mathcal{G}}_{\epsilon}$ among $w_{\epsilon} \in H^{1}\left(B_{\epsilon^{-1}}\right)$. This implies that $v_{\epsilon}$ minimizes $\mathcal{G} \epsilon(w)=\tilde{\mathcal{G}}_{\epsilon}(w)-\tilde{\mathcal{G}}_{\epsilon}(\phi)$ in the same class of functions.

(2) The sequence $\mathcal{G} \epsilon\left(v_{\epsilon}\right)$ is convergent.

(3) $v \in \mathcal{V}$.

(4) For every $w$ in $\mathcal{V}$ there is a sequence $w_{\epsilon}$ such that $w_{\epsilon} \in H^{1}\left(B_{\epsilon^{-1}}\right),\left.w_{\epsilon}\right|_{\partial B_{\epsilon^{-1}}}(x)=$ $\phi_{\epsilon}(x)$ and $\mathcal{G} \epsilon\left(w_{\epsilon}\right) \rightarrow \mathcal{G}(w)$.

(5) $\mathcal{G}_{\epsilon}\left(v_{\epsilon}\right) \rightarrow \mathcal{G}(v)$.

(6) Conclude the result using the previous steps.

Proof of Step (1): Notice first that for every $w_{\epsilon} \in H^{1}\left(B_{\epsilon^{-1}}\right)$ satisfying $\left.w_{\epsilon}\right|_{\partial B_{\epsilon}-1}=\phi(x)$ holds that $w_{\epsilon}^{\epsilon}(x)=w_{\epsilon}\left(\frac{x}{\epsilon}\right) \in H^{1}\left(B_{1}\right)$ and $\left.w_{\epsilon}^{\epsilon}\right|_{\partial B_{1}}=\phi_{\epsilon}(x)$. Recall that $u_{\epsilon}$ is a minimizer for $\mathcal{I}_{\epsilon}$ (defined by (16) $)$, that is for every $w_{\epsilon}^{\epsilon} \in H^{1}\left(B_{1}\right)$ satisfying $\left.w_{\epsilon}^{\epsilon}\right|_{\partial B_{1}}=\phi_{\epsilon}(x)$ holds

$$
\mathcal{I}_{\epsilon}\left(u_{\epsilon}\right) \leq \mathcal{I}_{\epsilon}\left(w_{\epsilon}^{\epsilon}\right)
$$

Dividing by $\epsilon$ and changing variables holds

$$
\frac{1}{\epsilon} \mathcal{I}_{\epsilon}\left(u_{\epsilon}\right)=\int_{B_{\frac{1}{\epsilon}}}\left(\left|D v_{\epsilon}\right|^{2}+W\left(v_{\epsilon}\right)\right) d y \leq \frac{1}{\epsilon} \mathcal{I}_{\epsilon}\left(w_{\epsilon}^{\epsilon}\right)=\int_{B_{\frac{1}{\epsilon}}}\left(\left|D w_{\epsilon}\right|^{2}+W\left(w_{\epsilon}\right)\right) d y
$$

or equivalently

$$
\tilde{\mathcal{G}}_{\epsilon}\left(v_{\epsilon}\right) \leq \tilde{\mathcal{G}}_{\epsilon}\left(w_{\epsilon}\right), \text { for every } w_{\epsilon} \in H^{1}\left(B_{\frac{1}{\epsilon}}\right)
$$

By subtracting $\tilde{\mathcal{G}}_{\epsilon}(\phi)$ we get

$$
\mathcal{G}_{\epsilon}\left(v_{\epsilon}\right) \leq \mathcal{G}_{\epsilon}\left(w_{\epsilon}\right) \text {, for every } w_{\epsilon} \in H^{1}\left(B_{\frac{1}{\epsilon}}\right)
$$

Proof of Step (2): Fix $0<\epsilon<\sigma$. We need to show that $\mathcal{G}_{\epsilon}\left(v_{\epsilon}\right)$ is a Cauchy sequence. Namely, we prove that for every $\delta>0$ there is an $\epsilon_{0}$ such that

$$
\left|\mathcal{G}_{\epsilon}\left(v_{\epsilon}\right)-\mathcal{G}_{\sigma}\left(v_{\sigma}\right)\right| \leq \delta \text { for } \epsilon, \sigma \leq \epsilon_{0} .
$$

We will study separately two cases: $\sigma \geq \sqrt{\epsilon}$ and $\sigma<\sqrt{\epsilon}$. 
- $\sigma \geq \sqrt{\epsilon}$

$$
\begin{aligned}
\left|\mathcal{G}_{\epsilon}\left(v_{\epsilon}\right)-\mathcal{G}_{\sigma}\left(v_{\sigma}\right)\right| \leq & \mid \int_{B_{\frac{1}{\epsilon}} \backslash B_{\frac{1}{\sigma}}}\left(\left|D v_{\epsilon}\right|^{2}-|D \phi|^{2}+W\left(v_{\epsilon}\right)-W(\phi)\right) d x \\
& +\int_{B_{\frac{1}{\sigma}}}\left(\left|D v_{\epsilon}\right|^{2}-\left|D v_{\sigma}\right|^{2}+W\left(v_{\epsilon}\right)-W\left(v_{\sigma}\right)\right) d x \mid \\
\leq & \int_{B_{\frac{1}{\epsilon}} \backslash B_{\frac{1}{\sqrt{\epsilon}}}}\left(\left.|| D v_{\epsilon}\right|^{2}-|D \phi|^{2}|+| W\left(v_{\epsilon}\right)-W(\phi) \mid\right) d x \\
& +\int_{B_{\frac{1}{\sqrt{\epsilon}}} \backslash B_{\frac{1}{\sigma}}}\left(\left|D v_{\epsilon}\right|^{2}-\left|D v_{\sqrt{\epsilon}}\right|^{2}|+| W\left(v_{\epsilon}\right)-W\left(v_{\sqrt{\epsilon}}\right) \mid\right) d x \\
& +\int_{B_{\frac{1}{\sqrt{\epsilon}}} \backslash B_{\frac{1}{\sigma}}}\left(\left|D v_{\sqrt{\epsilon}}\right|^{2}-|D \phi|^{2}|+| W\left(v_{\sqrt{\epsilon}}\right)-W(\phi) \mid\right) d x \\
& +\int_{B_{\frac{1}{\sigma}}}\left(\left.|| D v_{\epsilon}\right|^{2}-\left|D v_{\sigma}\right|^{2}|+| W\left(v_{\epsilon}\right)-W\left(v_{\sigma}\right) \mid\right) d x .
\end{aligned}
$$

Let $u_{\sigma}^{\epsilon}(x)=v_{\sigma}\left(\frac{x}{\epsilon}\right)$. Changing variables we have

$$
\begin{aligned}
\left|\mathcal{G}_{\epsilon}\left(v_{\epsilon}\right)-\mathcal{G}_{\sigma}\left(v_{\sigma}\right)\right| \leq & \int_{B_{1} \backslash B_{\sqrt{\epsilon}}}\left(\left.|| D u_{\epsilon}\right|^{2}-\left|D \phi_{\epsilon}\right|^{2}|+| \frac{W\left(u_{\epsilon}\right)-W\left(\phi_{\epsilon}\right)}{\epsilon^{2}} \mid\right) d x \\
& +\int_{B_{\sqrt{\epsilon} \backslash B_{\frac{\epsilon}{\sigma}}}}\left(\left.|| D u_{\epsilon}\right|^{2}-\left|D u_{\sqrt{\epsilon}}^{\epsilon}\right|^{2}|+| \frac{W\left(u_{\epsilon}\right)-W\left(u_{\sqrt{\epsilon}}^{\epsilon}\right)}{\epsilon^{2}} \mid\right) d x \\
& +\int_{B_{1} \backslash B_{\frac{\sqrt{\epsilon}}{\sigma}}}\left(\left.|| D u_{\sqrt{\epsilon}}\right|^{2}-\left|D \phi_{\sqrt{\epsilon}}\right|^{2}|+| \frac{W\left(u_{\sqrt{\epsilon}}\right)-W\left(\phi_{\sqrt{\epsilon}}\right)}{\epsilon} \mid\right) d x \\
& +\int_{B_{\frac{\epsilon}{\sigma}}}\left(\left.|| D u_{\epsilon}\right|^{2}-\left|D u_{\sigma}^{\epsilon}\right|^{2}|+| \frac{W\left(u_{\epsilon}\right)-W\left(u_{\sigma}\right)}{\epsilon^{2}} \mid\right) d x
\end{aligned}
$$

Notice that since $\sigma \geq \sqrt{\epsilon}$ we have that $\frac{\epsilon}{\sigma} \leq \sqrt{\epsilon}$. Then using Theorem 4.1 and Corollaries 4.2 and 4.3 we have that for every $m$ there is a constant, that depends on $m$, such that

$$
\left|\mathcal{G}_{\epsilon}\left(v_{\epsilon}\right)-\mathcal{G}_{\sigma}\left(v_{\sigma}\right)\right| \leq C \epsilon^{m} .
$$


- $\sigma \leq \sqrt{\epsilon}$

$$
\begin{aligned}
\left|\mathcal{G}_{\epsilon}\left(v_{\epsilon}\right)-\mathcal{G}_{\sigma}\left(v_{\sigma}\right)\right| \leq & \int_{B_{\frac{1}{\epsilon}} \backslash B_{\frac{1}{\sigma}}}\left(\left.|| D v_{\epsilon}\right|^{2}-|D \phi|^{2}|+| W\left(v_{\epsilon}\right)-W(\phi) \mid\right) d x \\
& +\int_{B_{\frac{1}{\sigma}}}\left(\left.|| D v_{\epsilon}\right|^{2}-\left|D v_{\sigma}\right|^{2}|+| W\left(v_{\epsilon}\right)-W\left(v_{\sigma}\right) \mid\right) d x \\
\leq & \int_{B_{\frac{1}{\epsilon}} \backslash B_{\frac{1}{\sigma}}}\left(\left.|| D v_{\epsilon}\right|^{2}-|D \phi|^{2}|+| W\left(v_{\epsilon}\right)-W(\phi) \mid\right) d x \\
& +\int_{B_{\frac{1}{\sigma}} \backslash B_{\frac{1}{\sqrt{\epsilon}}}}\left(\left.|| D v_{\epsilon}\right|^{2}-|D \phi|^{2}|+| W\left(v_{\epsilon}\right)-W(\phi) \mid\right) d x \\
& +\int_{B_{\frac{1}{\sigma}} \backslash B} \frac{1}{\sqrt{\epsilon}} \\
& +\int_{B_{\frac{1}{\sqrt{\epsilon}}}}\left(\left.|| D \phi\right|^{2}-\left|D v_{\sigma}\right|^{2}|+| W\left(v_{\sigma}\right)-W(\phi) \mid\right) d x
\end{aligned}
$$

Let $u_{\sigma}^{\epsilon}(x)=v_{\sigma}\left(\frac{x}{\epsilon}\right)$. Changing variables we have

$$
\begin{aligned}
\left|\mathcal{G}_{\epsilon}\left(v_{\epsilon}\right)-\mathcal{G}_{\sigma}\left(v_{\sigma}\right)\right| \leq & \int_{B_{1} \backslash B_{\sqrt{\epsilon}}}\left(\left|D u_{\epsilon}\right|^{2}-\left|D \phi_{\epsilon}\right|^{2}|+| \frac{W\left(u_{\epsilon}\right)-W\left(\phi_{\epsilon}\right)}{\epsilon^{2}} \mid\right) d x \\
& +\int_{B_{1} \backslash B_{\frac{\sigma}{\sqrt{\epsilon}}}}\left(\left.|| D u_{\sigma}\right|^{2}-\left|D \phi_{\sigma}\right|^{2}|+| \frac{W\left(u_{\sigma}\right)-W\left(\phi_{\sigma}\right)}{\sigma^{2}} \mid\right) d x \\
& +\int_{B_{\sqrt{\epsilon}}}\left(\left.|| D u_{\epsilon}\right|^{2}-\left|D u_{\sigma}^{\epsilon}\right|^{2}|+| \frac{W\left(u_{\epsilon}\right)-W\left(u_{\sigma}^{\epsilon}\right)}{\epsilon^{2}} \mid\right) d x
\end{aligned}
$$

Since $\sigma>\epsilon$, we have that $\frac{\sigma}{\sqrt{\epsilon}} \geq \sqrt{\sigma}$. Then, Theorem 4.1 and Corollaries 4.2 and 4.3 imply that for every $m$ there is a constant, that depend on $m$, such that

$$
\left|\mathcal{G}_{\epsilon}\left(v_{\epsilon}\right)-\mathcal{G}_{\sigma}\left(v_{\sigma}\right)\right| \leq C\left(\epsilon^{m}+\sigma^{m}\right) .
$$

We conclude from (98) and (99) that for every $m>0$ there is a constant $C$ such that

$$
\left|\mathcal{G}_{\epsilon}\left(v_{\epsilon}\right)-\mathcal{G}_{\sigma}\left(v_{\sigma}\right)\right| \leq C\left(\epsilon^{m}+\sigma^{m}\right) .
$$

Therefore $\mathcal{G}_{\epsilon}\left(v_{\epsilon}\right)$ is a Cauchy sequence of real numbers, thus convergent.

Proof of Step (3): Following the same method of the previous step we can prove the the sequences $\int_{B_{\frac{1}{\epsilon}}}\left|D v_{\epsilon}-D \phi\right|$ and $\int_{B_{\frac{1}{\epsilon}}}\left|v_{\epsilon}-\phi\right|$ are Cauchy sequences and therefore uniformly bounded. Fatou's Lemma implies that

$$
\begin{gathered}
\int_{\mathbb{R}^{2}}|D v-D \phi| d x \leq \int_{B_{\frac{1}{\epsilon}}}\left|D v_{\epsilon}-D \phi\right| d x<\infty \\
\int_{\mathbb{R}^{2}}|v-\phi| \leq \int_{B_{\frac{1}{\epsilon}}}\left|v_{\epsilon}-\phi\right| d x<\infty .
\end{gathered}
$$

That is $v \in \mathcal{V}$. 
Proof of Step (4): Consider a smooth function $\eta$ satisfying $\eta(x)=1$ for $|x| \leq \frac{1}{2}$ and $\eta(x)=0$ for $|x| \geq 1$. Define

$$
w_{\epsilon}(x)=\eta(\epsilon x) w(x)+(1-\eta(\epsilon x)) \phi .
$$

Then

Since $w \in \mathcal{V}$ we have

$$
\begin{aligned}
\left|\mathcal{G}_{\epsilon}\left(w_{\epsilon}\right)-\mathcal{G}(w)\right|= & \mid \int_{\mathbb{R}^{2} \backslash B_{\frac{1}{2 \epsilon}}}\left(|D w|^{2}-|D \phi|^{2}+W(w)-W(\phi)\right) d x \\
& -\int_{B_{\frac{1}{\epsilon} \backslash B_{\frac{1}{2 \epsilon}}}}\left(|\eta D w+(1-\eta) D \phi+D \eta(w-\phi)|^{2}-|D \phi|^{2}\right) d x \\
\leq & C\left|\int_{\mathbb{R}^{2} \backslash B_{\frac{1}{2 \epsilon}}}(|D w-D \phi|+|w-\phi|) d x\right| \\
& +\mid \int_{B_{\frac{1}{\epsilon} \backslash B_{\frac{1}{2 \epsilon}}}}(|\eta D w+(1-\eta) D \phi+D \eta(w-\phi)-D \phi| \\
\leq & \left|\int_{\mathbb{R}^{2} \backslash B_{\frac{1}{2 \epsilon}}}(|D w-D \phi|+|w-\phi|) d x\right| .
\end{aligned}
$$

$$
\lim _{\epsilon \rightarrow 0}\left|\mathcal{G}_{\epsilon}\left(w_{\epsilon}\right)-\mathcal{G}(w)\right|=0
$$

\section{Proof of Step (5)}

The previous step implies there is a $\tilde{v}_{\epsilon}$ such that

$$
\mathcal{G}_{\epsilon}\left(\tilde{v}_{\epsilon}\right) \rightarrow \mathcal{G}(v) .
$$

Since $v_{\epsilon}$ is a minimizer of $\mathcal{G}_{\epsilon}$ we have that

$$
\mathcal{G}_{\epsilon}\left(v_{\epsilon}\right) \leq \mathcal{G}_{\epsilon}\left(\tilde{v}_{\epsilon}\right) .
$$

Taking limits when $\epsilon \rightarrow 0$ we have

$$
\lim _{\epsilon \rightarrow 0} \mathcal{G}\left(v_{\epsilon}\right) \leq \mathcal{G}(v)
$$

In particular, $\mathcal{G}(v)$ is bounded below. Fatou's Lemma allow us to conclude the other inequality:

$$
\mathcal{G}(v) \leq \lim _{\epsilon \rightarrow 0} \mathcal{G}\left(v_{\epsilon}\right)
$$

Proof of Step (6) Consider $w \in \mathcal{V}$, then take $w_{\epsilon}$ as in step (44). Then the minimality of $v_{\epsilon}$ implies

$$
\mathcal{G}_{\epsilon}\left(v_{\epsilon}\right) \leq \mathcal{G}\left(w_{\epsilon}\right)
$$

Taking limits as $\epsilon \rightarrow 0$ we conclude that

$$
\mathcal{G}(v) \leq \mathcal{G}(w)
$$

which finishes the proof. 


\section{APPENDIX}

In this appendix we present a collection of technical results used in the previous sections.

We start by stating some results about the Heat Kernel, used mainly in Section 4 . Consider a ball $B \subset \mathbb{R}^{2}$. Then $\mathcal{H}_{B}$ can be described as follows:

$$
\begin{aligned}
\left(\frac{d}{d t}-\Delta_{x}\right) \mathcal{H}_{B}(x, y, t) & =0, \\
\mathcal{H}_{B}(x, y, t) & =0 \text { whenever } x \in \partial B, \\
\lim _{t \rightarrow 0^{+}} \mathcal{H}_{B}(x, y, t) & =\delta_{y}(x) .
\end{aligned}
$$

Hence, the solution to the equation

$$
\begin{aligned}
\left(\frac{d}{d t}-\Delta_{x}\right) u(x, t) & =f(x, t), \\
u(x, t) & =0 \text { whenever } x \in \partial B, \\
u(x, 0) & =g(x),
\end{aligned}
$$

can be represented as

$$
u(x, t)=\int_{0}^{t} \int_{B} \mathcal{H}_{B}(x, y, t-s) f(y, s) d y d s+\int_{B} \mathcal{H}_{B}(x, y, t) g(y) d y .
$$

We will use this representation to prove the following lemmmas. Let us define $P$ to be the heat operator, that is

$$
P u=\frac{d}{d t} u-\Delta u
$$

First we prove some bounds over $\mathcal{H}_{B}$ :

Lemma A-1. It holds that

$$
0 \leq \int_{B} \mathcal{H}_{B}(x, y, t-s) d y d s \leq 1
$$

$$
0 \leq \int_{s}^{t} \int_{B} \mathcal{H}_{B}(x, y, t-s) d y d s \leq(t-s) .
$$

Proof. The proof follows by maximum principle. Notice that the single-valued function

satisfies the equation

$$
v(x, t)=\int_{B} \mathcal{H}_{B}(x, y, t-s) d y d s
$$

$$
\begin{aligned}
P v(x, t) & =0, \\
v(x, t) & =0 \text { whenever } x \in \partial B, \\
v(x, s) & =1 .
\end{aligned}
$$

Since the function 0 is a sub-solution to $(\mathrm{A}-8)-(\mathrm{A}-9)-(\mathrm{A}-10)$ we have that

$$
0 \leq v(x, t) .
$$

Similarly, the function 1 is a super-solution. Hence,

$$
v(x, t) \leq 1
$$


which proves (A-6). Equation (A-7) follows by integrating inequality (A-6).

We also include without proof the following theorem (see [10, [14 for example).

Theorem A-1. (Theorem 3.1 in [10]) Let $\mathcal{M}$ be a $n$ dimensional compact Riemannian manifold with boundary. Then there is a Dirichlet heat kernel, that is a function

$$
\mathcal{H} \in C^{\infty}(\mathcal{M} \times \mathcal{M} \times(0, \infty)) .
$$

satisfying (A-1)- (A-3)- (A-2).

The smoothness of $\mathcal{H}(x, x, t)$ may be described as follows

$$
\mathcal{H}(x, x, t)=t^{-\frac{n}{2}}(A(x, t)+B(x, t))
$$

with $A \in C^{\infty}(\mathcal{M} \times[0, \infty))$ and $B$ is supported near the boundary, where in local coordinates $\left(x^{\prime}, x_{n}\right) \in U^{\prime} \times[0, \tilde{\delta}) \subset \mathcal{M}, \quad U^{\prime} \subset \mathbb{R}^{n-1}$ open, one has

$$
B(x, t)=b\left(x^{\prime}, \frac{x_{n}}{\sqrt{t}}, t\right), b \in C^{\infty}\left(u^{\prime} \times \mathbb{R}_{+} \times[0, \infty)_{\sqrt{t}}\right)
$$

with $b\left(x^{\prime}, \psi_{n}, t\right)$ rapidly decaying as $\psi_{n} \rightarrow \infty$.

Now we devote ourselves to prove Lemma 4.1. We start with the following a priori bound:

Theorem A-2. Let $\tilde{h}_{\epsilon}(x, t): \mathbb{R}^{2} \rightarrow \mathbb{R}^{2}$ satisfy

$$
\begin{aligned}
P \tilde{h}_{\epsilon}+\frac{\nabla_{v} W\left(\tilde{h}_{\epsilon}\right)}{2} & =0 \text { for } x \in B_{\frac{1}{\epsilon}} \\
\left.\tilde{h}_{\epsilon}(x, t)\right|_{\partial B_{\frac{1}{\epsilon}}} & =\phi(x) \\
\tilde{h}_{\epsilon}(x, 0) & =\psi_{\epsilon}(x),
\end{aligned}
$$

where $W: \mathbb{R}^{2} \rightarrow \mathbb{R}$ is a proper $\mathcal{C}^{2}$ function, bounded below, with a finite number of critical points (denoted by $\left\{c_{i}\right\}_{i=1}^{m}$ ), and such that the Hessian of $W(u)$ is positive semidefinite for $|u| \geq K$, where $K$ is a fixed real number. Then if $\tilde{h}_{\epsilon}(x, 0)=\psi_{\epsilon}(x)$ is bounded there is a constant $C$ that depends only on $W, \phi$ and $\psi_{\epsilon}$ such that $\left|\tilde{h}_{\epsilon}(x, t)\right| \leq C$.

Proof. Consider $l_{\epsilon}(x, t)=W\left(\tilde{h}_{\epsilon}\right)(x, t)$; then

$$
\begin{aligned}
\left(l_{\epsilon}\right)_{t}-\Delta l_{\epsilon} & =\nabla_{v} W\left(\tilde{h}_{\epsilon}\right) \cdot\left(\tilde{h}_{\epsilon}\right)_{t}-\sum_{i}\left(\nabla_{v} W\left(\tilde{h}_{\epsilon}\right) \cdot\left(\tilde{h}_{\epsilon}\right)_{x_{i}}\right)_{x_{i}} \\
& =\nabla_{v} W\left(\tilde{h}_{\epsilon}\right) \cdot\left(\tilde{h}_{\epsilon}\right)_{t}-\left(W^{\prime \prime}\left(\tilde{h}_{\epsilon}\right) \nabla \tilde{h}_{\epsilon}\right) \cdot \nabla \tilde{h}_{\epsilon}-\nabla_{v} W\left(\tilde{h}_{\epsilon}\right) \cdot \Delta \tilde{h}_{\epsilon}
\end{aligned}
$$

where $W^{\prime \prime}$ denotes the Hessian matrix of $W$. Since $\tilde{h}_{\epsilon}$ satisfies (A-11), this becomes

$$
\left(l_{\epsilon}\right)_{t}-\Delta l_{\epsilon}+\frac{\left|W^{\prime}\left(\tilde{h}_{\epsilon}\right)\right|^{2}}{2}+\left(W^{\prime \prime}\left(\tilde{h}_{\epsilon}\right) \nabla u\right) \cdot \nabla \tilde{h}_{\epsilon}=0
$$

We are going to find bounds over $l_{\epsilon}$ at the boundary of $B_{\frac{1}{\epsilon}}$ and over its possible interior maxima in terms of $\max \phi, K, W\left(c_{i}\right)$ and $\max W(\psi(x))$.

Since for every $|x|=1$ holds $\tilde{h}_{\epsilon}(x, t)=\phi(x)$ and $\phi$ is uniformly bounded, we have that

$$
l_{\epsilon}(x) \leq \max W(\phi(x)) \text { for every } x \in \partial B_{\frac{1}{\epsilon}} \text {. }
$$


Suppose that $l_{\epsilon}$ has an interior maximum at $\left(x_{0}, t_{0}\right)$ and $\left|\tilde{h}_{\epsilon}\left(x_{0}, t_{0}\right)\right| \geq K$. Since $\left(x_{0}, t_{0}\right)$ is a maximum for $l_{\epsilon}$, it holds that $\left(l_{\epsilon}\right)_{t}\left(x_{0}, t_{0}\right) \geq 0$ and $\Delta l_{\epsilon}\left(x_{0}, t_{0}\right) \leq 0$. We also have by hypothesis that $W^{\prime \prime}(u)$ is positive semidefinite for $|u| \geq K$, hence

$$
\left(l_{\epsilon}\right)_{t}-\Delta l_{\epsilon}+\frac{\left|\nabla{ }_{u} W\left(\tilde{h}_{\epsilon}\right)\right|^{2}}{2}+\left(W^{\prime \prime}\left(\tilde{h}_{\epsilon}\right) \nabla \tilde{h}_{\epsilon}\right) \cdot \nabla \tilde{h}_{\epsilon} \geq 0 .
$$

The inequality is strict (which contradicts $(\underline{\mathrm{A}-14}))$ unless $\frac{\left|\nabla_{u} W\left(\tilde{h}_{\epsilon}\right)\right|^{2}}{\epsilon^{2}}=\left(W^{\prime \prime}\left(\tilde{h}_{\epsilon}\right) \nabla \tilde{h}_{\epsilon}\right) \cdot \nabla \tilde{h}_{\epsilon}=$ 0 . If $\nabla_{v} W\left(\tilde{h}_{\epsilon}\left(x_{0}, t_{0}\right)\right)=0$, we would have $\tilde{h}_{\epsilon}\left(x_{0}, t_{0}\right)=c_{i}$ for some $i$, therefore $W\left(\tilde{h}_{\epsilon}(x, t)\right) \leq$ $W\left(c_{i}\right)$. From this and the previous computations we conclude that $l_{\epsilon}$ is uniformly bounded.

Since $W$ is a proper function, we have that there is a constant $C$ such that

$$
\left|\tilde{h}_{\epsilon}(x)\right| \leq C \text { for } x \in \bar{B}_{\frac{1}{\epsilon}},
$$

which finishes the proof

By observing that solutions to (46)-(47)-(48) can be written as $h_{\epsilon}(x, t)=\tilde{h}_{\epsilon}(x, t)-V_{\vec{q}}(x)$, where $\tilde{h}_{\epsilon}$ is a solution to (A-11)-(A-12) - (A-13) we have

Corollary A-1. Let $h_{\vec{q}}(x, t): B_{\frac{1}{\epsilon}} \rightarrow \mathbb{R}^{2}$ be a solution to (46)-(47)- 48 , where $W: \mathbb{R}^{2} \rightarrow \mathbb{R}$ is a proper $\mathcal{C}^{2}$ function, bounded below, with a finite number of critical points and such that the Hessian of $W(w)$ is positive semidefinite for $|w| \geq K$, where $K$ is a fixed real number. Then if $h_{\vec{q}}(x, 0)=\psi_{\epsilon}(x)$ is bounded there is a constant $C$ that depends only on $W, \phi, U_{\vec{q}}$ and $\psi_{\epsilon}$ such that $\left|h_{\vec{q}}(x, t)\right| \leq C$.

\section{Proof of Lemma 4.1 Let}

$$
\mathcal{C}_{\left[\bar{t}_{1}, \bar{t}_{2}\right]}(B)=\left\{w: \bar{B} \times\left[\bar{t}_{1}, \bar{t}_{2}\right] \rightarrow \mathbb{R}^{2}: w \text { is a uniformly bounded continuous function }\right\}
$$

with the standard sup norm.

Consider some $\tau \geq 0$ and define $F_{\vec{q}}^{\tau}\left(\cdot, \psi_{\vec{q}}^{\tau}\right): \mathcal{C}_{\left[\tau, \tau+\frac{2 \beta}{M}\right]}\left(B_{\epsilon^{-1}}\right) \rightarrow \mathcal{C}_{\left[\tau, \tau+\frac{2 \beta}{M}\right]}\left(B_{\epsilon^{-1}}\right)$ by

$$
\begin{gathered}
F_{\vec{q}}^{\tau}\left(w, \psi_{\vec{q}}^{\tau}\right)(x, t)=\int_{\tau}^{t} \int_{B_{\epsilon^{-1}}} \mathcal{H}_{B_{\epsilon^{-1}}}(x, y, t-s)\left(\frac{-W^{\prime}\left(w(y, s)+V_{\vec{q}}\right)}{2}+\Delta V_{\vec{q}}(y)\right) d y d s \\
\quad+\int_{B_{\epsilon^{-1}}} \mathcal{H}_{B_{\epsilon^{-1}}}(x, y, t) \psi_{\vec{q}}^{\tau}(y) d y
\end{gathered}
$$

Notice that Duhamel's formula implies that fixed points of the function $F_{\vec{q}}^{\tau}\left(\cdot, \psi_{\vec{q}}^{\tau}\right)$ are solutions to (46) in $\left[\tau, \tau+\frac{2 \beta}{M}\right]$. Hence, in order to prove Lemma 4.1 we will use the following strategy: For every $\tau, \psi^{\tau}$ and appropriate constants $\beta, M$ we find a fixed point of $F_{\vec{q}}^{\tau}\left(\cdot, \psi_{\vec{q}}^{\tau}\right)$ in some appropriate space; then we choose $\psi^{\tau}$ appropriately so the fixed points (that were found in the previous step) "glue" together appropriately; we finish by showing that in fact (46) holds in the whole domain, as well as (47) and (48).

Claim. If there is a constant $M$ such that $\left|W^{\prime \prime}\right| \leq M$ and $\psi_{\vec{q}}^{\tau}$ is uniformly bounded, then $F_{\vec{q}}^{\tau}\left(\cdot, \psi_{\vec{q}}^{\tau}\right): \mathcal{C}_{\left[\tau, \tau+\frac{2 \beta}{M}\right]}\left(B_{\epsilon^{-1}}\right) \rightarrow \mathcal{C}_{\left[\tau, \tau+\frac{2 \beta}{M}\right]}\left(B_{\epsilon^{-1}}\right)$ is well defined for each $\vec{q} \in \mathcal{Q}$, where $\mathcal{Q}$ is given by (45). If additionally for any given $\tau$ and $\beta \in(0,1)$ we have that $\bar{t}$ satisfies $|\bar{t}-\tau| \leq \frac{2 \beta}{M}$, then $F_{\vec{q}}^{\tau}\left(\cdot, \psi_{\vec{q}}^{\tau}\right)$ is a contraction mapping with constant $\beta$ in $\mathcal{C}_{\left[\tau, \tau+\frac{2 \beta}{M}\right]}\left(B_{\epsilon^{-1}}\right)$. 
To prove that the function $F_{\vec{q}}^{\tau}\left(\cdot, \psi_{\vec{q}}^{\tau}\right): \mathcal{C}_{\left[\tau, \tau+\frac{2 \beta}{M}\right]}\left(B_{\epsilon^{-1}}\right) \rightarrow \mathcal{C}_{\left[\tau, \tau+\frac{2 \beta}{M}\right]}\left(B_{\epsilon^{-1}}\right)$ is well defined we need to show that $F_{\vec{q}}^{\tau}\left(\cdot, \psi_{\vec{q}}^{\tau}\right)$ maps any uniformly bounded function into a uniformly bounded function, that is for any function $w$ that satisfies $|w(x, t)| \leq C$ for all $(x, t) \in B_{\epsilon^{-1}} \times[\tau, \bar{t}]$ it holds that $\left|F_{\vec{q}}^{\tau}\left(w, \psi_{\vec{q}}^{\tau}\right)(x, t)\right| \leq \bar{C}$ for all $(x, t) \in B_{\epsilon^{-1}} \times[\tau, \bar{t}]$.

By continuity of $W^{\prime}$ we have that if $\sup _{B_{\epsilon}-1 \times[\tau, t]}|w(x, t)| \leq C$ then there is a constant $C_{1}$ such that $\sup _{(x, t) \in B_{\epsilon^{-1}} \times[\tau, \nexists]}\left|W^{\prime}(w)(x, t)\right| \leq C_{1}$. Using the definition of $V_{\vec{q}}$, we can also find constants $C_{2}$ and $C_{3}$ that

$$
\left|\Delta V_{\vec{q}}\right| \leq C_{2}
$$

and

This implies

$$
\left|V_{\vec{q}}\right| \leq C_{3}
$$

$$
\begin{aligned}
\left|F_{\vec{q}}^{\tau}\left(w, \psi_{\vec{q}}^{\tau}\right)\right|(x, t) & \\
\leq & \left(C_{1}+C_{2}\right) \int_{\tau}^{\bar{t}} \int_{B_{\epsilon^{-1}}} \mathcal{H}_{B_{\epsilon^{-1}}}(x, y, t-\tau-s) d y d s \\
& +\sup _{x \in B_{\epsilon^{-1}}}\left|\psi_{\epsilon}^{\tau}(x)\right| \int_{B_{\epsilon^{-1}}} \mathcal{H}_{B_{\epsilon^{-1}}}(x, y, t-\tau) d y+C_{3} \\
\leq & \left(C_{1}+C_{2}\right)(\bar{t}-\tau)+\sup _{x \in B_{\epsilon^{-1}}}\left|\psi_{\epsilon}^{\tau}\right|(x)+C_{3}=\bar{C}<\infty
\end{aligned}
$$

for all $(x, t)$. Hence $F_{\vec{q}}^{\tau}\left(\cdot, \psi_{\vec{q}}^{\tau}\right)$ is well defined for each $\vec{q} \in \mathcal{Q}$ (where $\mathcal{Q}$ is given by (45)).

Now we show that if $|\bar{t}-\tau| \leq \frac{2 \beta}{M}$, then $F_{\vec{q}}^{\tau}\left(\cdot, \psi_{\vec{q}}^{\tau}\right)$ is a contraction mapping.

Since $\left|W^{\prime \prime}\right| \leq M$ we have that

$$
\left|W^{\prime}\left(w_{1}\right)-W^{\prime}\left(w_{2}\right)\right| \leq M\left|w_{1}-w_{2}\right| .
$$

Then for every $x \in B_{\epsilon^{-1}}$ and $t \in[\tau, \bar{t}]$ it holds that

$$
\begin{gathered}
\left|F_{\vec{q}}^{\tau}\left(w_{1}, \psi_{\vec{q}}^{\tau}\right)-F_{\vec{q}}^{\tau}\left(w_{2}, p s i_{\vec{q}}^{\tau}\right)\right|(x, t)=\left|\int_{\tau}^{t} \int_{B_{\epsilon^{-1}}} \mathcal{H}_{B_{\epsilon^{-1}}}(x, y, t-s-\tau) \frac{-W^{\prime}\left(w_{1}(y, s)\right)+W^{\prime}\left(w_{2}(y, s)\right)}{2} d y d s\right| \\
\leq \frac{M(\bar{t}-\tau)}{2} \sup _{(x, t) \in B_{\epsilon^{-1}} \times[\tau, \bar{t}]}\left|w_{1}-w_{2}\right|(x, t) .
\end{gathered}
$$

Then for $|\bar{t}-\tau| \leq \frac{2 \beta}{M}$ holds

$$
\sup _{(x, t) \in B_{\epsilon^{-1}} \times[\tau, \bar{t}]}\left|F_{\vec{q}}^{\tau}\left(w_{1}, \psi_{\vec{q}}^{\tau}\right)-F_{\vec{q}}^{\tau}\left(w_{2}, \psi_{\vec{q}}^{\tau}\right)\right|(x, t) \leq \beta \sup _{(x, t) \in B_{\epsilon^{-1}} \times[\tau, \bar{t}]}\left|w_{1}-w_{2}\right|(x, t)
$$

and $F_{\vec{q}}^{\tau}\left(\cdot, \psi_{\vec{q}}^{\tau}\right): B_{\epsilon^{-1}} \times\left[\tau, \tau+\frac{2 \beta}{M}\right] \rightarrow B_{\epsilon^{-1}} \times\left[\tau, \tau+\frac{2 \beta}{M}\right]$ is a contraction with constant $\beta$.

We will assume that $\left|W^{\prime \prime}\right| \leq M$ and at the end of the proof we will point out the necessary modifications in the general case. Fix $\beta<1$ and let

$$
\begin{aligned}
\tau_{i} & =i \frac{2 \beta}{M} \\
\bar{t}_{i} & =\tau_{i+1},
\end{aligned}
$$




$$
F_{\vec{q}, i}=F_{\vec{q}}^{\tau}\left(\cdot, \psi_{\vec{q}}^{\tau}\right)
$$

with $i=0, \ldots, I_{\beta}$, where the constant $\beta, I_{\beta} \in \mathbb{N}$ satisfy $\frac{T M}{2 \beta} \leq I_{\beta} \leq 2 \frac{\bar{t} M}{2 \beta}$. By the definition of $\tau_{i}, \bar{t}_{i}$ we have that $\bar{t}_{I_{\beta}} \geq \bar{t}$. We will redefine $\bar{t}_{I_{\beta}}=\bar{t}$.

By the previous claim $F_{\vec{q}, i}$ is contraction, hence it has a unique fixed point, $h_{\vec{q}}^{i}$. That is

$$
F_{\vec{q}, i}\left(h_{\vec{q}}^{i}(x, t)\right)=h_{\vec{q}}^{i}(x, t) .
$$

Moreover, since this this fixed point is bounded we have that $F_{\vec{q}}^{\tau}\left(h_{\vec{q}}^{i}, \psi_{\vec{q}}^{\tau}\right) \in C^{1, \frac{1}{2}}\left(B_{\epsilon^{-1}} \times\right.$ $\left.\left(\tau_{i}, \tau_{i+1}\right]\right)$. Recursively, $h_{\vec{q}}^{i} \in C^{\infty}$. From (ㅅ-19) and Duhamel's formula we can conclude that (46) and (47) hold for $t \in\left[\tau_{i}, \bar{t}_{i}\right]$. We also have

$$
h_{\vec{q}}^{i}\left(x, \tau_{i}\right)=\psi_{\vec{q}}^{\tau_{i}}(x)
$$

for $(x, t) \in B_{\epsilon^{-1}} \times\left(\tau_{i}, \bar{t}_{i}\right)$.

Now define recursively $\psi_{\vec{q}}^{\tau_{i}}(x)$ :

$$
\begin{gathered}
\psi_{\vec{q}}^{\tau_{0}}(x)=\psi_{\vec{q}}(x) \\
\psi_{\vec{q}}^{\tau_{i}}(x)=h_{\vec{q}}^{i-1}\left(x, \tau_{i}\right) .
\end{gathered}
$$

Then $h_{\vec{q}}(x, t)$ defined by

$$
h_{\vec{q}}(x, t)=h_{\vec{q}}^{i}(x, t) \text { for } t \in\left[\tau_{i}, \bar{t}_{i}\right]
$$

satisfies (46) for $t \neq \tau_{i}$. Moreover, by writing

$$
\begin{aligned}
h_{\vec{q}}^{i+1}(x, t)=\int_{\bar{t}_{i}}^{t} \int_{B_{\epsilon^{-1}}} & \mathcal{H}_{B_{\epsilon^{-1}}}\left(x, y, t-\bar{t}_{i}-s\right) \frac{-W^{\prime}\left(h_{\vec{q}}^{i+1}+V_{\vec{q}}\right)(y, s)}{2} d y d s \\
& +\int_{B_{\epsilon^{-1}}} \mathcal{H}_{B_{\epsilon^{-1}}}\left(x, y, t-\bar{t}_{i}\right) h_{\vec{q}}^{i}\left(y, \bar{t}_{i}\right) d y
\end{aligned}
$$

standard computations show that $h_{\vec{q}}$ satisfies (46) for every $t$. Since $h_{\vec{q}}$ also satisfies (47)(48) we have that $h_{\vec{q}}$ is the desired solution. In particular, this implies that $h_{\vec{q}}$ is the fixed point of $F_{\vec{q}}$. Uniqueness follows from the fact that fixed points of contraction mappings are unique.

In order to prove equation (49) we observe that since $h_{\vec{q}}$ is a fixed point of $F_{\vec{q}}^{\tau}$, standard computations imply for any function $w_{\vec{q}}$

$$
\begin{aligned}
\left|h_{\vec{q}}-w_{\vec{q}}\right| & \leq \frac{1}{1-\beta} \sup _{B_{\epsilon^{-1}} \times\left[\tau, \tau+\frac{2 \beta}{M}\right]}\left|F_{\vec{q}}^{\tau}\left(w_{\vec{q}}\right)-w_{\vec{q}}\right| \\
& \leq \frac{1}{1-\beta}\left(\sup _{B_{\epsilon^{-1}} \times\left[\tau, \tau+\frac{2 \beta}{M}\right]}\left|F_{\vec{q}}^{\tau}\left(w_{\vec{q}}\right)-F_{\vec{q}}\left(w_{\vec{q}}\right)\right|+\sup _{B_{\epsilon^{-1}} \times\left[\tau, \tau+\frac{2 \beta}{M}\right]}\left|F_{\vec{q}}\left(w_{\vec{q}}\right)-w_{\vec{q}}\right|\right) .
\end{aligned}
$$

The definitions of $F_{\vec{q}}^{\tau}$ and $F_{\vec{q}}$ imply that

$$
P\left(F_{\vec{q}}^{\tau}\left(w_{\vec{q}}\right)-F_{\vec{q}}\left(w_{\vec{q}}\right)\right)=\frac{\nabla_{v} W\left(w_{\vec{q}}\right)}{2}-\frac{\nabla_{v} W\left(w_{\vec{q}}\right)}{2}=0
$$

and

$$
F_{\vec{q}}^{\tau}\left(w_{\vec{q}}\right)(x, \tau)-F_{\vec{q}}\left(w_{\vec{q}}\right)(x, \tau)=h_{\vec{q}}(x, \tau)-F_{\vec{q}}\left(w_{\vec{q}}\right)(x, \tau) .
$$


Using Duhamel's formula we have

$$
F_{\vec{q}}^{\tau}\left(w_{\vec{q}}\right)-F_{\vec{q}}\left(w_{\vec{q}}\right)=\int_{B_{\epsilon^{-1}}} \mathcal{H}_{B_{\epsilon^{-1}}}(x, y, t-\tau)\left(h_{\vec{q}}(y, \tau)-F_{\vec{q}}\left(w_{\vec{q}}\right)(y, \tau)\right) d y .
$$

Together with Lemma A-1, this implies

$$
\sup _{B_{\epsilon^{-1}} \times\left[\tau, \tau+\frac{2 \beta}{M}\right]}\left|F_{\vec{q}}^{\tau}\left(w_{\vec{q}}\right)-F_{\vec{q}}\left(w_{\vec{q}}\right)\right| \leq \sup _{B_{\epsilon^{-1}}}\left|h_{\vec{q}}(x, \tau)-F_{\vec{q}}\left(w_{\vec{q}}\right)\right|(x, \tau) .
$$

Using (A-24) we conclude inequality (49)

$$
\left|h_{\vec{q}}-w_{\vec{q}}\right| \leq \frac{1}{1-\beta}\left(2 \sup _{B_{\epsilon^{-1}} \times\left[\tau, \tau+\frac{2 \beta}{M}\right]}\left|F_{\vec{q}}\left(w_{\vec{q}}\right)-w_{\vec{q}}\right|+\sup _{B_{\epsilon^{-1}}}\left|h_{\vec{q}}-w_{\vec{q}}\right|(x, \tau)\right)
$$

For the general case (that is when there is no constant $M$ such that $\left|W^{\prime \prime}\right| \leq M$ ) we fix $K>0$ large enough. Then we replace $W$ for a function $\tilde{W}$ that satisfies:

- there is an $M$ such that $\left|\tilde{W}^{\prime \prime}\right| \leq M$,

- $\tilde{W}(u)=W(u)$ for $u \leq \max \{2 C, K\}$, where $C$ is the constant given by Theorem A-2,

- $\tilde{W}$ has the same critical points as $W$.

Then, our previous computations imply that there is a unique solution $h_{\vec{q}}$ to

$$
\begin{aligned}
P h_{\vec{q}}+\frac{\nabla_{v} \tilde{W}\left(h_{\vec{q}}+V_{\vec{q}}\right)}{2}+\Delta V_{\vec{q}} & =0 \\
h_{\vec{q}}(x) & =0 \text { for every } x \in \partial B_{\epsilon^{-1}} \\
h_{\vec{q}}(x, 0) & =\psi_{\epsilon}(x) .
\end{aligned}
$$

Moreover for $w_{\vec{q}}$ as in the hypothesis holds

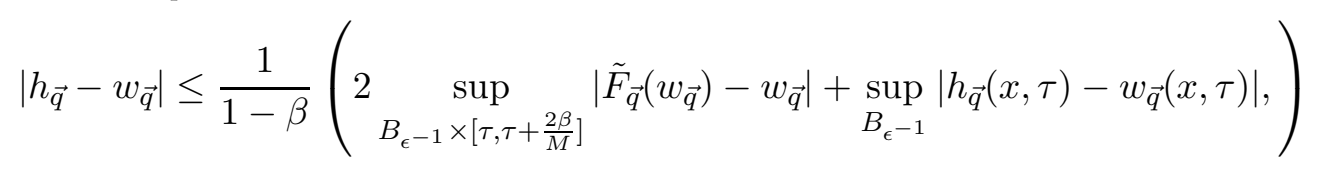

where $\tilde{F}_{\vec{q}}$ is analogous to $F_{\vec{q}}$ substituting $W$ for $\tilde{W}$.

However, following the proof Theorem $\overline{\mathrm{A}-2}$ we also have that $\left|h_{\vec{q}}\right|(x, t) \leq C$, where $C$ is the constant given by Theorem A-2, This fact and the construction of $\tilde{W}$ imply that $h_{\epsilon}$ is not only a solution to (A-25)-(A-26)-(A-27), but also to (46)-(47)-(48) (since within this range $W=\tilde{W})$. Moreover, for $w_{\vec{q}}$ satisfying $\left|w_{\vec{q}}\right| \leq K$ we will have $\tilde{F}_{\vec{q}}\left(w_{\vec{q}}\right)-w_{\vec{q}}=F_{\vec{q}}\left(w_{\vec{q}}\right)-w_{\vec{q}}$, concluding that (49) holds and finishing the proof of the Theorem.

Theorem A-3. Let $h_{\vec{q}}$ be a solution to (46)- (47)-(48), then there is a constant $K$, independent of $\vec{q}$, such that for every $x \in B_{\frac{1}{\epsilon}}$

$$
\left|D h_{\vec{q}}\right| \leq K
$$

Proof. Recall that $h_{\vec{q}}$ is vector-valued. We will denote the coordinate $i$-th of the vector $h_{\vec{q}}$ by $h_{\vec{q}}^{i}$ and, similarly, $\left(\nabla W\left(h_{\vec{q}}\right)\right)^{i}$ is the the $i$ th coordinate of $\nabla W\left(h_{\vec{q}}\right)$. We are going to prove separately that for each coordinate that there is a constant $C_{i}$ such that $\left|\nabla h_{\vec{q}}^{i}\right| \leq C_{i}$.

Let $f:\{(x, y): y \geq 0\} \rightarrow B_{\frac{1}{\epsilon}}$ be defined by

$$
f(x, y)=\frac{1}{\epsilon}\left(\frac{x^{2}+y^{2}-1}{x^{2}+(y+1)^{2}}, \frac{-2 x}{x^{2}+(y+1)^{2}}\right) .
$$


In complex number notation, we can write for $z=x+i y$

$$
f(z)=\frac{z-i}{z+i}
$$

Define

$$
\left.s_{\vec{q}}^{i}(x, y, t)=h_{\vec{q}}^{i}(f(x, y), t)\right) .
$$

It satisfies

$$
\begin{aligned}
\frac{8}{\epsilon\left(x^{2}+(y+1)^{2}\right)} \frac{d s_{\vec{q}}^{i}}{d t}-\Delta s_{\vec{q}}^{i} & =-\frac{8}{\epsilon\left(x^{2}+(y+1)^{2}\right)}\left(\nabla W\left(h_{\vec{q}}\right)\right)^{i}+\Delta v^{i} \text { for } x \in \mathbb{R}, y>0 \\
s_{\vec{q}}^{i}(x, y, t) & =0 \text { for } y=0 \text { or }|(x, y)| \rightarrow \infty \\
s_{\vec{q}}(x, y, 0) & =0 .
\end{aligned}
$$

Let $\tilde{P}$ be the operator defined by

$$
\tilde{P} u=\frac{8}{\epsilon\left(x^{2}+(y+1)^{2}\right)} \frac{d u}{d t}-\Delta u .
$$

Theorem A-2 and the definition of $s_{\vec{q}}^{i}$ implies that there is a constant $C$ independent of $\epsilon$ such that

Moreover,

$$
\tilde{P} s_{\vec{q}}^{i} \leq \frac{C}{\epsilon}
$$

$$
\frac{\partial s_{\vec{q}}^{i}}{\partial y}(x, 0)=0
$$

Now define

$$
w_{\vec{q}}^{i}(x, y, t)=s_{\vec{q}}^{i}(x, y, t)-\frac{C}{\epsilon}\left(y^{2}+y\right)
$$

Then

$$
\begin{aligned}
\tilde{P} w_{\vec{q}}^{i} & =\tilde{P} s_{\vec{q}}^{i}-2 \frac{C}{\epsilon} \leq 0, \\
w_{\vec{q}}^{i}(x, 0, t) & =0 \text { for every } x \in \mathbb{R}^{2} \text { and } t>0, \\
w_{\vec{q}}^{i}(x, y, 0) & <0 \text { for }|(x, y)| \rightarrow \infty \text { and } y>0 .
\end{aligned}
$$

Also,

$$
\frac{\partial w_{\vec{q}}^{i}}{\partial y}(x, y, 0)=-\frac{C}{\epsilon}(2 y+1) \leq 0 .
$$

Claim. The maximum of $w_{\vec{q}}^{i}$ cannot be attained in the interior.

If the max is attained at some point in the interior, must hold that $\Delta w_{\vec{q}}^{i}<0$ and $\frac{d w_{\vec{q}}^{i}}{d t} \geq 0$. Hence $\tilde{P} w_{\vec{q}}^{i} \geq 0$, which is a contradiction and finishes the proof of the claim.

Since the maximum is attained on the boundary it must be attained at $y=0$. Therefore

$$
\frac{\partial w_{\vec{q}}^{i}}{\partial y}(x, y, t) \leq 0 \text { for every } t
$$


This implies that

$$
\frac{\partial s_{\vec{q}}^{i}}{\partial y}(x, y, t) \leq \frac{C}{\epsilon}(2 y+1) .
$$

This procedure can be repeated for $-s_{\vec{q}}^{i}$, concluding that

$$
\left|\frac{\partial s_{\vec{q}}^{i}}{\partial y}(x, y, t)\right| \leq \frac{C}{\epsilon}(2 y+1) .
$$

Since the inverse function of $f$ is

$$
f^{-1}(w)=\frac{1+\epsilon w}{1-\epsilon w}
$$

using (A-30), (A-29) and (A-31) we have (in complex number notation) for any $w \in B_{\frac{1}{\epsilon}}$ that

$$
\left|\nabla h_{\vec{q}}^{i}(w, t) \cdot(1-\epsilon w)^{2}\right| \leq 2 C\left(\frac{1-\epsilon^{2}|w|^{2}}{1+\epsilon^{2}|w|^{2}-\epsilon(w+\bar{w})}+\epsilon\right),
$$

where $\bar{w}$ is the conjugate of $w$.

Similarly, if we define (by performing a rotation of $f$ ):

$$
g(z)=\frac{i}{\epsilon} \frac{z-i}{z+i}
$$

and

$$
r(x, y, t)=h_{\vec{q}}(g(x, y), t),
$$

following the same method we obtain

$$
\left|\nabla h_{\vec{q}}^{i}(w, t) \cdot i(1+i \epsilon w)^{2}\right| \leq 2 C\left(\frac{1-\epsilon^{2}|w|^{2}}{1+\epsilon^{2}|w|^{2}+i \epsilon(w-\bar{w})}+\epsilon\right) .
$$

Notice for $w$ away from $\frac{1}{\epsilon}$ and $\frac{i}{\epsilon}$ holds that $i(1+i \epsilon w)^{2}$ and $(1-\epsilon w)^{2}$ are linearly independent as vectors in $\mathbb{R}^{2}$. Fixing some $\delta$ small enough and considering $w$ such that $\left|w-\frac{i}{\epsilon}\right| \geq \delta$ and $\left|w-\frac{1}{\epsilon}\right| \geq \delta$ we have that $\frac{1-\epsilon^{2}|w|^{2}}{1+\epsilon^{2}|w|^{2}-\epsilon(w+\bar{w})}+\epsilon$ and $\frac{1-\epsilon^{2}|w|^{2}}{1+\epsilon^{2}|w|^{2}+i \epsilon(w-\bar{w})}+\epsilon$ are bounded above and below independent of $\epsilon$. Hence

$$
\left|\nabla h_{\vec{q}}^{i}(w, t)\right| \leq C \text { for every }\left|w-\frac{i}{\epsilon}\right| \geq \delta,\left|w-\frac{1}{\epsilon}\right| \geq \delta .
$$

Now considering rotation of $f$ of $\pi$ and $\frac{3}{2} \pi$ radians (that is $\tilde{f}(z)=-\frac{1}{\epsilon} \frac{z-i}{z+i}$ and $\tilde{g}(z)=$ $\left.-\frac{i}{\epsilon} \frac{z-i}{z+i}\right)$ and following the same procedure we fund bounds for $\left|\nabla h_{\vec{q}}^{i}(w, t)\right|$ near $\frac{1}{\epsilon}$ and $\frac{i}{\epsilon}$, concluding the proof.

Similarly it follows

Corollary A-2. Let $k_{\vec{q}}$ be defined by (56). Then there is a constant $K$, independent of $\vec{q}$, such that for every $x \in B_{1}$

$$
\left|D k_{\vec{q}}\right| \leq \frac{K}{\epsilon}
$$




\section{REFERENCES}

[1] Nicholas D. Alikakos, Santiago I. Betelú, and Xinfu Chen. Explicit stationary solutions in multiple well dynamics and non-uniqueness of interfacial energy densities. European J. Appl. Math., 17(5):525-556, 2006.

[2] S. Allen and J. Cahn. A microscopic theory for antiphase boundary motion ands its application to antiphase domain coarsening. Acta Metal, 27:1085-1095, 1979.

[3] Sisto Baldo. Minimal interface criterion for phase transitions in mixtures of Cahn-Hilliard fluids. Ann. Inst. H. Poincaré Anal. Non Linéaire, 7(2):67-90, 1990.

[4] Fabrice Bethuel, Haïm Brezis, and Frédéric Hélein. Asymptotics for the minimization of a GinzburgLandau functional. Calc. Var. Partial Differential Equations, 1(2):123-148, 1993.

[5] Fabrice Bethuel, Haïm Brezis, and Frédéric Hélein. Ginzburg-Landau vortices. Progress in Nonlinear Differential Equations and their Applications, 13. Birkhäuser Boston Inc., Boston, MA, 1994.

[6] Lia Bronsard, Changfeng Gui, and Michelle Schatzman. A three-layered minimizer in $\mathbf{R}^{2}$ for a variational problem with a symmetric three-well potential. Comm. Pure Appl. Math., 49(7):677-715, 1996.

[7] Lia Bronsard and Fernando Reitich. On three-phase boundary motion and the singular limit of a vectorvalued Ginzburg-Landau equation. Arch. Rational Mech. Anal., 124(4):355-379, 1993.

[8] Gilberto Flores and Pablo Padilla and Yoshihiro Tonegawa. Higher energy solutions in the theory of phase transitions: a variational approach, J. Differential Equations 169:190-207, 2001.

[9] Enrico Giusti. Minimal surfaces and functions of bounded variation, volume 80 of Monographs in Mathematics. Birkhäuser Verlag, Basel, 1984.

[10] Daniel Grieser. Notes on the heat kernel asymptotics.

[11] Symmetric quadruple phase transitions Changfeng Gui and Michelle Schatzman Indiana Univ. Math. J., 57: 781-836. 2008.

[12] Tom Ilmanen. Convergence of the Allen-Cahn equation to Brakke's motion by mean curvature. J. Differential Geom., 38(2):417-461, 1993.

[13] Robert V. Kohn and Peter Sternberg. Local minimisers and singular perturbations. Proc. Roy. Soc. Edinburgh Sect. A, 111(1-2):69-84, 1989.

[14] Richard B. Melrose. The Atiyah-Patodi-Singer index theorem, volume 4 of Research Notes in Mathematics. A K Peters Ltd., Wellesley, MA, 1993.

[15] W. W. Mullins. Two-dimensional motion of idealized grain boundaries. J. Appl. Phys., 27:900-904, 1956.

[16] W. W. Mullins. Two-dimensional motion of idealized grain boundaries [ MR0078836 (17,1252g)]. In Fundamental contributions to the continuum theory of evolving phase interfaces in solids, pages 70-74. Springer, Berlin, 1999.

[17] Frank Pacard and Tristan Rivière. Linear and nonlinear aspects of vortices. Progress in Nonlinear Differential Equations and their Applications, 39. Birkhäuser Boston Inc., Boston, MA, 2000. The Ginzburg-Landau model.

[18] Jacob Rubinstein, Peter Sternberg, and Joseph B. Keller. Fast reaction, slow diffusion, and curve shortening. SIAM J. Appl. Math., 49(1):116-133, 1989.

[19] Mariel Sáez. Relaxation of the curve shortening flow via the parabolic Ginzburg-Landau equation. PhD thesis, Stanford University, 2005.

[20] Mariel Sáez Trumper. Relaxation of the curve shortening flow via the parabolic ginzburg-landau equation. To appear in Calc. of Var., 2007.

[21] Mariel Sáez Trumper. Relaxation of the flow of triods via the vector-valued parabolic Allen-Cahn equation. To appear in J. Reine Angew. Math. (Crelle's). .

[22] Peter Sternberg. Vector-valued local minimizers of nonconvex variational problems. Rocky Mountain J. Math., 21(2):799-807, 1991.

[23] Peter Sternberg and William P. Zeimer. Local minimisers of a three-phase partition problem with triple junctions. Proc. Roy. Soc. Edinburgh Sect. A, 124(6):1059-1073, 1994. 
Mariel Saez Trumper

Max Planck Institute for Gravitational Physics

Albert Einstein Institute

Am Mühlenberg 1, D-14476 Golm, Germany,

Germany .

E-mail address: mariel.saez@aei.mpg.de 